ALEA, Lat. Am. J. Probab. Math. Stat. 16, 1029-1054 (2019)

\title{
Coalescing directed random walks on the backbone of a $1+1$-dimensional oriented percolation cluster converge to the Brownian web
}

\author{
Matthias Birkner, Nina Gantert and Sebastian Steiber \\ Johannes Gutenberg University Mainz, Institute for Mathematics \\ Staudingerweg 9, 55099 Mainz, Germany \\ E-mail address: birkner@mathematik.uni-mainz.de \\ Technical University Munich, Fakultät für Mathematik \\ Boltzmannstraße 3, 85748 Garching, Germany \\ E-mail address: gantert@ma.tum.de \\ Johannes Gutenberg University Mainz, Institute for Mathematics \\ Staudingerweg 9, 55099 Mainz, Germany \\ E-mail address: sebastian.steiber@gmail.com
}

\begin{abstract}
We consider the backbone of the infinite cluster generated by supercritical oriented site percolation in dimension $1+1$. A directed random walk on this backbone can be seen as an "ancestral lineage" of an individual sampled in the stationary discrete-time contact process. Such ancestral lineages were investigated in Birkner et al. (2013) where a central limit theorem for a single walker was proved. Here, we consider infinitely many coalescing walkers on the same backbone starting at each space-time point. We show that, after diffusive rescaling, the collection of paths converges in distribution (under the averaged law) to the Brownian web. Hence, we prove convergence to the Brownian web for a particular system of coalescing random walks in a dynamical random environment. An important tool in the proof is a tail bound on the meeting time of two walkers on the backbone, started at the same time. Our result can be interpreted as an averaging statement about the percolation cluster: apart from a change of variance, it behaves as the full lattice, i.e. the effect of the "holes" in the cluster vanishes on a large scale.
\end{abstract}

\section{Introduction}

Informally, the Brownian web is a system of one-dimensional coalescing Brownian motions starting from every point in space and time. It was first introduced in Arratia (1979), studied rigorously in Tóth and Werner (1998), Fontes et al. (2004)

Received by the editors December 8th, 2018; accepted August 30th, 2019. 2010 Mathematics Subject Classification. 60J70, 82C22, 60K35, 60K37.

Key words and phrases. Oriented percolation, coalescing random walks, Brownian web. 
and has since then been shown to be a scaling limit of many 1+1-dimensional coalescing structures. See also Schertzer et al. (2017) for an overview, historical discussion and references. Possibly the most natural example that comes to mind in this respect is the system of coalescing random walks on $\mathbb{Z}$ which is dual to the one-dimensional voter model (see, e.g., Liggett, 1999). This was shown to converge to the Brownian web (in Fontes et al., 2004 for the nearest neighbor case and in Newman et al. (2005) in the general case). One often interprets the voter model as a population model in which there is always exactly one individual at each site $x \in \mathbb{Z}$, which can be of one of two possible types, say. The dual system of random walks is then naturally interpreted as ancestral lines of the individuals. Note that while the total population is infinite, the local population size at a site in the voter model is fixed (at one).

There is interest in spatial population models with randomly fluctuating local population sizes, see, e.g., Etheridge (2004), Fournier and Méléard (2004), Etheridge (2006), Birkner et al. (2016) and the discussion and references there. In this case, ancestral lines are random walks in a dynamic random environment which is given by the time reversal of the population model. Birkner et al. (2013) considered the specific but prototypic example of the stationary supercritical discrete time contact process. Its time-reversal is the backbone of the supercritical oriented percolation cluster and in Birkner et al. (2013), a central limit theorem was proved for such a walk, i.e., for a single ancestral lineage. It is then a natural problem to study the joint behavior of several or in fact of all ancestral lineages, hence a system of coalescing random walks in a dynamic random environment. We address this problem here in the case $d=1$. Our main result, Theorem 1.1 below, shows then that on large scales, the effect of the local population fluctuations manifests itself only as a scaling factor compared to the case of fixed local sizes. This in a sense rigorously confirms the approach that is often taken in modelling spatially distributed biological populations where one exogenously fixes the local population size by considering so-called stepping stone models, see, e.g., Kimura (1953), Wilkinson-Herbots (1998). See also Section 3 below for more details on the relation to the discrete time contact process and also Birkner et al. (2016) for discussion and a broader class of examples.

1.1. Set-up. Let $\omega:=\{\omega(x, n):(x, n) \in \mathbb{Z} \times \mathbb{Z}\}$ be i.i.d. Bernoulli $(p)$ random variables. A space-time site $(x, n) \in \mathbb{Z} \times \mathbb{Z}$ is said to be open if $\omega(x, n)=1$ and closed if $\omega(x, n)=0$. A directed open path from $(x, m)$ to $(y, n)$ for $m \leqslant n$ is a sequence $x_{m}, \ldots, x_{n}$ such that $x_{m}=x, x_{n}=y,\left|x_{k}-x_{k-1}\right| \leqslant 1$ for $k=m+1, \ldots, n$ and $\omega\left(x_{k}, k\right)=1$ for all $k=m, \ldots, n$. We write $(x, m) \stackrel{\omega}{\rightarrow}(y, n)$ if such an open path exists and $(x, m) \stackrel{\omega}{\rightarrow} \infty$ if there exists at least one infinite directed open path starting at $(x, m)$.

There is $p_{c}=p_{c}(1) \in(0,1)$ such that $\mathbb{P}((0,0) \stackrel{\omega}{\rightarrow} \infty)>0$ if and only if $p>p_{c}$ (see e.g. Theorem 1 in Grimmett and Hiemer, 2002). We assume from now on that $p>p_{c}$. Let

$$
\begin{aligned}
\mathcal{C} & :=\{(x, n) \in \mathbb{Z} \times \mathbb{Z}:(x, n) \stackrel{\omega}{\rightarrow} \infty\} \\
& =\{(x, n) \in \mathbb{Z} \times \mathbb{Z}: \forall k>n \exists y \in \mathbb{Z} \text { such that }(x, n) \stackrel{\omega}{\rightarrow}(y, k)\}
\end{aligned}
$$

be the backbone of the space-time cluster of oriented percolation (note that $\mathcal{C}$ is a function of $\omega$ and $|\mathcal{C}|=\infty$ a.s. for $p>p_{c}$ ). 
We consider walks $X^{\left(x_{0}, t_{0}\right)}=\left(X_{t}^{\left(x_{0}, t_{0}\right)}\right)_{t \in \mathbb{Z}, t \geqslant t_{0}}$ starting at any space-time point $\left(x_{0}, t_{0}\right) \in \mathbb{Z} \times \mathbb{Z}$ and moving as directed simple random walk on $\mathcal{C}$. More precisely, let

$$
U(x):=\{y \in \mathbb{Z}:|x-y| \leqslant 1\}
$$

be the $\ell_{\infty}$-neighbourhood of site $x \in \mathbb{Z}$ and let $\widetilde{\omega}=\left(\widetilde{\omega}(x, n): x \in \mathbb{Z}^{d}, n \in \mathbb{Z}\right)$, where $\widetilde{\omega}(x, n)=(\widetilde{\omega}(x, n)[1], \widetilde{\omega}(x, n)[2], \widetilde{\omega}(x, n)[3])$ is a uniformly chosen permutation of $U(x)$, independently distributed for different $(x, n)$ 's and independent of the $\omega$ 's. Define

$\Phi(x, n):= \begin{cases}\widetilde{\omega}(x, n)[\min \{i:(\widetilde{\omega}(x, n)[i], n+1) \in \mathcal{C}\}], & \text { if } \mathcal{C} \cap(U(x) \times\{n+1\}) \neq \varnothing, \\ \widetilde{\omega}(x, n)[1], & \text { otherwise }\end{cases}$

Note that when $(x, n) \in \mathcal{C}$, the first case occurs and $\Phi(x, n)$ is a uniform pick among those sites in $\{y:(y, n+1) \in \mathcal{C}\}$, the $n+1$-time slice of $\mathcal{C}$; when $(x, n) \notin \mathcal{C}, \Phi(x, n)$ is simply a uniformly chosen neighbour of $x$. We put

$$
X_{t_{0}}^{\left(x_{0}, t_{0}\right)}:=x_{0}, \quad X_{t+1}^{\left(x_{0}, t_{0}\right)}:=\Phi\left(X_{t}^{\left(x_{0}, t_{0}\right)}, t\right), t \in \mathbb{Z}_{+} .
$$

For fixed $\left(x_{0}, t_{0}\right) \in \mathbb{Z} \times \mathbb{Z}$, given $\omega, X^{\left(x_{0}, t_{0}\right)}$ is a (time-inhomogeneous) Markov chain with

$$
\begin{aligned}
& P_{\omega}\left(X_{t+1}^{\left(x_{0}, t_{0}\right)}=y \mid X_{t}^{\left(x_{0}, t_{0}\right)}=x\right) \\
& =\mathbb{1}_{U(x)}(y) \times \begin{cases}|(U(x) \times\{t+1\}) \cap \mathcal{C}|^{-1} & \text { if }(y, t+1) \in \mathcal{C}, \\
0 & \text { if }(y, t+1) \notin \mathcal{C} \text { but } \\
|U(x)|^{-1} & (U(x) \times\{t+1\}) \cap \mathcal{C} \neq \varnothing,\end{cases} \\
& \text { if }(U(x) \times\{t+1\}) \cap \mathcal{C}=\varnothing
\end{aligned}
$$

and $P_{\omega}\left(X_{t_{0}}^{\left(x_{0}, t_{0}\right)}=x_{0}\right)=1$. In fact, (1.4) implements a (coalescing) stochastic flow with individual paths having transition probabilities given by (1.5).

When $t_{0}=0$ is fixed, we will abbreviate $X^{(z)}:=X^{(z, 0)}$ for $z \in \mathbb{Z}$.

This walk was introduced and studied in Birkner et al. (2013), we refer to that paper for a more thorough discussion of the background and related works. In particular, Birkner et al. (2013) describe a regeneration construction for $X^{\left(x_{0}, t_{0}\right)}$ and derived a LLN and a quenched CLT from it, see Theorems 1.1 and 1.3 there; the results also imply that $X^{\left(x_{0}, t_{0}\right)}$ and $X^{\left(x_{1}, t_{0}\right)}$ are "almost independent" when they are far apart. We recall in Section 2.1 below some details from Birkner et al. (2013) that are relevant for the present study, see in particular (2.3) for the non-trivial variance in the CLT. Thus, we expect that on sufficiently large space-time scales, any collection $X^{\left(x_{0}, t_{0}\right)}, X^{\left(x_{1}, t_{1}\right)}, \ldots, X^{\left(x_{n}, t_{n}\right)}$ should look similar to (coalescing) random walks.

Remark 1.1. The study of random walks in dynamic random environments is currently a very active field which we cannot survey completely here, see e.g. Avena et al. (2011), Hilário et al. (2015), Bethuelsen and Völlering (2016), Salvi and Simenhaus (2018) and the references there for recent examples. We note however that the walks we consider here are somewhat unusual with respect to that literature because of the time directions: There, one often considers scenarios where both the walk and the random environment have the same "natural" forwards in time direction as a (Markov) process whereas in our case, forwards in time for the walk 
means backwards in time for the environment, namely the discrete time contact process. More precisely, the "time-slices" of the cluster $\mathcal{C}$ can be seen to be equal in distribution to the time-reversal of a stationary discrete-time contact process $\left(\eta_{n}\right)$, we refer to Birkner et al. (2013) for details.

1.2. Main result: Brownian web limit in $d=1$. Before stating our main result we briefly recall a suitable definition of the Brownian web, following for example Fontes et al. (2004) or Sun (2005). See also Schertzer et al. (2017) for a broader introduction and an overview of related work. We define a metric on $\mathbb{R}^{2}$ by

$$
\rho\left(\left(x_{1}, t_{1}\right),\left(x_{2}, t_{2}\right)\right):=\left|\tanh \left(t_{1}\right)-\tanh \left(t_{2}\right)\right| \vee\left|\frac{\tanh \left(x_{1}\right)}{1+\left|t_{1}\right|}-\frac{\tanh \left(x_{2}\right)}{1+\left|t_{2}\right|}\right|
$$

Let $R_{c}^{2}$ be the completion of $\mathbb{R}^{2}$ under $\rho$. We can think of $R_{c}^{2}$ as the image of $[-\infty, \infty] \times[-\infty, \infty]$ under the mapping

$$
(x, t) \mapsto\left(\frac{\tanh (x)}{1+|t|}, \tanh (t)\right) \in R_{c}^{2},
$$

i.e., $R_{c}^{2}$ can be identified with the square $[-1,1] \times[-1,1]$ where the line $[-1,1] \times\{1\}$ and the line $[-1,1] \times\{-1\}$ are squeezed to two single points which we call $(*, \infty)$ and $(*,-\infty)$.

We define $\Pi$ to be the set of functions $f:[\sigma, \infty] \longrightarrow[-\infty, \infty]$ with "starting points" $\sigma \in[-\infty, \infty]$, such that the mapping $t \mapsto(f(\sigma \vee t), t)$ from $(\mathbb{R},|\cdot|)$ to $\left(R_{c}^{2}, \rho\right)$ is continuous. We consider the elements in $\Pi$ as a tuple of the function $f$ and its starting point $\sigma$. The set $\Pi$ together with the metric

$d\left((f, \sigma),\left(g, \sigma^{\prime}\right)\right):=\left|\tanh (\sigma)-\tanh \left(\sigma^{\prime}\right)\right| \vee \sup _{t \geqslant \sigma \wedge \sigma^{\prime}}\left|\frac{\tanh (f(t \vee \sigma))}{1+|t|}-\frac{\tanh \left(g\left(t \vee \sigma^{\prime}\right)\right)}{1+|t|}\right|$

becomes a complete separable metric space. Let $\mathcal{H}$ be the set of compact subsets of $(\Pi, d)$. Equipped with the Hausdorff metric

$d_{\mathcal{H}}\left(K_{1}, K_{2}\right):=\sup _{(f, \sigma) \in K_{1}} \inf _{\left(g, \sigma^{\prime}\right) \in K_{2}} d\left((f, \sigma),\left(g, \sigma^{\prime}\right)\right) \vee \sup _{\left(g, \sigma^{\prime}\right) \in K_{2}} \inf _{(f, \sigma) \in K_{1}} d\left((f, \sigma),\left(g, \sigma^{\prime}\right)\right)$,

$\mathcal{H}$ is a complete separable metric space. Let $\mathcal{B}_{\mathcal{H}}$ be the Borel $\sigma$-algebra associated with the metric $d_{\mathcal{H}}$. We can characterize the Brownian web (BW) as follows:

Definition 1.2 (Brownian web). The Brownian web is a $\left(\mathcal{H}, \mathcal{B}_{\mathcal{H}}\right)$-valued random variable $\mathcal{W}$, whose distribution is uniquely determined by the following properties:

(i) For each deterministic $z \in \mathbb{R}^{2}$, the set $\mathcal{W}(z):=\{(f, \sigma) \in \mathcal{W}:(f(\sigma), \sigma)=z\}$ contains exactly one element almost surely.

(ii) For all $z_{1}, \ldots, z_{k} \in \mathbb{R}^{2},\left(\mathcal{W}\left(z_{1}\right), \ldots, \mathcal{W}\left(z_{k}\right)\right)$ is distributed as coalescing Brownian motions.

(iii) For any countable and dense subset $D$ of $\mathbb{R}^{2}$, almost surely, $\mathcal{W}$ is the closure of $\{\mathcal{W}(z): z \in D\}$ in $(\Pi, d)$.

Let us give a precise definition of the system of coalescing random walks starting from each point contained in the space-time-cluster of oriented percolation: Let $\mathcal{C}=\{(x, n) \in \mathbb{Z} \times \mathbb{Z}:(x, n) \stackrel{\omega}{\rightarrow} \infty\}$ be the set of all points in the space-time lattice 
which are connected to infinity (as defined in (1.1)). If a space-time point $z=$ $(x, n) \in \mathbb{Z} \times \mathbb{Z}$ is in $\mathcal{C}$ let

$\pi^{z}$ be the linearly interpolated path of the random walk $X^{z}$

starting from $z$ with dynamics (1.4).

If a point $z \in \mathbb{Z} \times \mathbb{Z}$ is not in $\mathcal{C}$, we choose the next point to the left of $z$ that is connected to infinity and define $\pi^{z}$ as a linearly interpolated copy of the path starting there. In formulas, if $z=(x, n) \notin \mathcal{C}$ we define

$$
c((x, n)):=(\max \{y \leqslant x:(y, n) \in \mathcal{C}\}, n) \text { and }\left(\pi^{z}(t)\right)_{t \geqslant n}:=\left(\pi^{c(z)}(t)\right)_{t \geqslant n} .
$$

Let $\boldsymbol{\Gamma}$ be the collection of all paths, i.e.

$$
\Gamma:=\left\{\pi^{z}: z \in \mathbb{Z} \times \mathbb{Z}\right\}=\left\{\pi^{z}: z \in \mathcal{C}\right\} .
$$

Since all paths in $\boldsymbol{\Gamma}$ are equicontinuous the closure of $\boldsymbol{\Gamma}$, which we also denote by $\boldsymbol{\Gamma}$, is a random variable taking values in $\left(\mathcal{H}, \mathcal{B}_{\mathcal{H}}\right)$.

In order to formulate the convergence theorem precisely we consider for $\delta>0$ and $b>0$ ( $b$ normalizes the standard deviation) the diffusive scaling map

$$
S_{b, \delta}:=\left(S_{b, \delta}^{1}, S_{b, \delta}^{2}\right):\left(R_{c}^{2}, d\right) \longrightarrow\left(R_{c}^{2}, d\right),
$$

where

$$
S_{b, \delta}(x, t):=\left(S_{b, \delta}^{1}(x, t), S_{b, \delta}^{2}(t)\right):= \begin{cases}\left(\frac{x \delta}{b}, \delta^{2} t\right), & \text { if }(x, t) \in \mathbb{R}^{2} \\ \left( \pm \infty, \delta^{2} t\right), & \text { if }(x, t)=( \pm \infty, t), t \in \mathbb{R}, \\ (*, \pm \infty), & \text { if }(x, t)=(*, \pm \infty) .\end{cases}
$$

The mapping $S_{b, \delta}$ is naturally extended to $(\Pi, d)$ via

$$
\begin{aligned}
S_{b, \delta}: \Pi & \longrightarrow \Pi \\
(\pi, t) & \mapsto\left(S_{b, \delta}^{1} \circ \pi, S_{b, \delta}^{2} \circ t\right) .
\end{aligned}
$$

For $K \subset \Pi$ we set $S_{b, \delta} K:=\left\{S_{b, \delta}((\pi, t)):(\pi, t) \in K\right\}$. Note that $K \in \mathcal{H}$ implies $S_{b, \delta} K \in \mathcal{H}$.

Theorem 1.0 (Birkner et al. (2013)). There is $v \in(0, \infty)$ such that conditioned on $(0,0) \in \mathcal{C}$,

$$
S_{v, \delta} \pi^{(0,0)} \underset{\delta \downarrow}{\stackrel{d}{\longrightarrow}} \text { standard Brownian motion. }
$$

The variance $v$ has a description in terms of regeneration times, which we recall from Birkner et al. (2013) in (2.3) below (cf. Birkner et al. (2013, Remark 1.2)).

Our main result is the following theorem.

Theorem 1.1. The $\left(\mathcal{H}, \mathcal{B}_{\mathcal{H}}\right)$-valued random variables $\left(S_{v, \delta} \boldsymbol{\Gamma}\right)$ converge in distribution to the Brownian web as $\delta \downarrow 0$.

Remark 1.2. 1. An analogous result holds when $U(x)=\{y:|y-x| \leqslant R\}$ for some $R \in \mathbb{N}$. Furthermore, note that even for $R=1$, paths in $\boldsymbol{\Gamma}$ can cross each other without coalescing.

2. In the parlance of random walks in random environments, Theorems 1.0 and 1.1 are annealed limit theorems, i.e., the randomness refers to jointly averaging the walk and the realization of the percolation cluster. In fact, Birkner et al. (2013) proved also a quenched version of Theorem 1.0, where a typical cluster is fixed and 
randomness refers only to the steps of the walk. However, we presently do not have a quenched analogue of Theorem 1.1 (see also the discussion in Section 3 below).

3. Sarkar and Sun (2013) considered the system of rightmost paths on an oriented (bond) percolation cluster and showed that it converges to the Brownian web after suitable centering and rescaling. Thus, in Sarkar and Sun (2013), walkers move to the right whenever possible (and in particular they cannot cross each other) whereas in our set-up, the walks pick uniformly among the allowed neighbors.

We prove Theorem 1.1 in Section 2 and discuss some implications and further questions in Section 3.

\section{Proofs}

Remark 2.1. In the proofs that follow $C$ and $c$ denote some positive constants whose exact value is not important for the argument. The constants $C$ and $c$ may also vary within a chain of inequalities. If the value of a certain constant is important for a later step, we add a subscript to it $C_{1}, C_{2}, \ldots$

2.1. Preliminaries. Here, we briefly recall concepts and results from Birkner et al. (2013) that will be required for our arguments.

For $z=(x, n) \in \mathbb{Z} \times \mathbb{Z}$, writing $B_{z}:=\{z \in \mathcal{C}\}$, we abbreviate

$$
\widetilde{\mathbb{P}}_{z}(\cdot):=\mathbb{P}\left(\cdot \mid B_{z}\right) \quad \text { and } \quad \widetilde{\mathbb{P}}_{z_{1}, z_{2}}(\cdot):=\mathbb{P}\left(\cdot \mid B_{z_{1}} \cap B_{z_{2}}\right) .
$$

Birkner et al. (2013, Sections 2.1-2.2) describes a regeneration construction for $\left(X_{t}^{z}\right)_{t \geqslant 0}$ : There are random times $0=T_{0}^{z}<T_{1}^{z}<T_{2}^{z}<\ldots$ such that with $Y_{i}^{z}:=X_{T_{i}^{z}}^{z}-X_{T_{i-1}^{z}}^{z}$, the sequence of space-time increments along the regeneration times $T_{i}^{z}$

$$
\left(Y_{i}^{z}, T_{i}^{z}-T_{i-1}^{z}\right)_{i=1,2, \ldots} \quad \text { is i.i.d. under } \mathbb{P}\left(\cdot \mid B_{z}\right)
$$

with $\widetilde{\mathbb{P}}_{z}\left(\left|Y_{1}^{z}\right| \geqslant n\right), \widetilde{\mathbb{P}}_{z}\left(T_{1}^{z}-T_{0}^{z} \geqslant n\right) \leqslant C e^{-c n}$ (see Birkner et al. (2013, Lemma 2.5)). By symmetry, $\widetilde{\mathbb{E}}_{z}\left[Y_{1}^{z}\right]=0$. In fact, (2.2) already yields an (annealed) central limit theorem with limit variance

$$
v=\frac{\widetilde{\mathbb{E}}_{z}\left[\left(Y_{1}^{z}\right)^{2}\right]}{\widetilde{\mathbb{E}}_{z}\left[T_{1}^{z}\right]} \in(0, \infty)
$$

see Birkner et al. (2013, Remark 1.2). (In Birkner et al., 2013, all this is formulated for $z=(0,0)$ but by shift-invariance of the joint distribution of $\omega$ and $\widetilde{\omega}$, it holds for any $z \in \mathbb{Z} \times \mathbb{Z}$ and in particular $v$ in (2.3) does not depend on $z$.)

For $z_{1}=\left(x_{1}, 0\right), z_{2}=\left(x_{2}, 0\right) \in \mathbb{Z} \times \mathbb{Z}$ consider the simultaneous regeneration times $0=T_{0}^{\mathrm{sim}}<T_{1}^{\mathrm{sim}}<T_{2}^{\mathrm{sim}}<\ldots$ for $X^{z_{1}}$ and $X^{z_{2}}$, defined via

$$
\left\{T_{i}^{\text {sim }}: i \in \mathbb{N}_{0}\right\}=\left\{T_{j}^{z_{1}}: j \in \mathbb{N}_{0}\right\} \cap\left\{T_{j^{\prime}}^{z_{2}}: j^{\prime} \in \mathbb{N}_{0}\right\}
$$

(we have $T_{i}^{\text {sim }}<\infty$ a.s. for all $i$, see Birkner et al., 2013, Section 3.1). In our notation we suppress the dependence of $T_{i}^{\mathrm{sim}}$ on the starting points $z_{1}, z_{2}$.

Write $\hat{X}_{\ell}^{z_{1}}:=X_{T_{\ell}^{\text {sim }}}^{z_{1}}, \hat{X}_{\ell}^{z_{2}}:=X_{T_{\ell}^{\text {sim }}}^{z_{2}}, \ell \in \mathbb{N}_{0}$. In Birkner et al. (2013, Section 3.1) it is shown (with a slightly different notation, see also Birkner et al. (2013, Remark 3.3)) that the sequence of pairs of path increments between the simultaneous 
regeneration times,

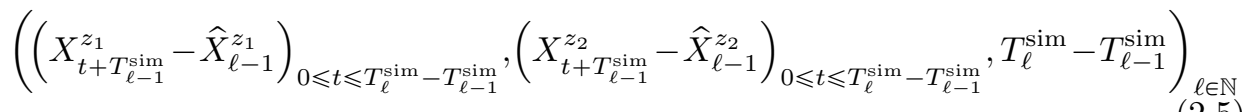

forms a Markov chain under $\widetilde{\mathbb{P}}_{z_{1}, z_{2}}$, see Birkner et al. (2013, Lemma 3.2). Furthermore, the transition probabilities depend only on the current positions $\left(\hat{X}_{\ell}^{z_{1}}, \hat{X}_{\ell}^{z_{2}}\right)$ and the increments between simultaneous regeneration times have uniformly exponentially bounded tails,

$\widetilde{\mathbb{P}}_{z_{1}, z_{2}}\left(T_{\ell}^{\mathrm{sim}}-T_{\ell-1}^{\mathrm{sim}}>n\right) \leqslant C e^{-c n}$ for all $n \in \mathbb{N}, z_{1}=\left(x_{1}, 0\right), z_{2}=\left(x_{2}, 0\right) \in \mathbb{Z} \times \mathbb{Z}$

(see Birkner et al., 2013, Lemma 3.1). In particular, $\left(\hat{X}_{\ell}^{z_{1}}, \hat{X}_{\ell}^{z_{2}}\right)_{\ell \in \mathbb{N}_{0}}$ is itself a Markov chain under $\widetilde{\mathbb{P}}_{z_{1}, z_{2}}$ and - by shift invariance of the joint distribution of $\omega$ and $\widetilde{\omega}$ - its transition matrix is invariant under simultaneous shifts in both coordinates, i.e.

$$
\begin{aligned}
& \widetilde{\mathbb{P}}_{z_{1}, z_{2}}\left(\hat{X}_{\ell+1}^{z_{1}}=y_{1}^{\prime}, \hat{X}_{\ell+1}^{z_{2}}=y_{2}^{\prime} \mid \hat{X}_{\ell}^{z_{1}}=y_{1}, \hat{X}_{\ell}^{z_{2}}=y_{2}\right) \\
& =\widetilde{\mathbb{P}}_{z_{1}, z_{2}}\left(\hat{X}_{\ell+1}^{z_{1}}=y_{1}^{\prime}+y, \hat{X}_{\ell+1}^{z_{2}}=y_{2}^{\prime}+y \mid \hat{X}_{\ell}^{z_{1}}=y_{1}+y, \hat{X}_{\ell}^{z_{2}}=y_{2}+y\right)
\end{aligned}
$$

for all $y_{1}, y_{1}^{\prime}, y_{2}, y_{2}^{\prime}, y \in \mathbb{Z}$. Thus $\widehat{D}_{\ell}^{z_{1}, z_{2}}:=\widehat{X}_{\ell}^{z_{1}}-\widehat{X}_{\ell}^{z_{2}}$, the difference of the two walks along simultaneous regeneration times, forms also a Markov chain; we denote its transition matrix by $\widehat{\Psi}_{\text {diff }}^{\text {joint }}$ (in the notation of Birkner et al., 2013, Lemma 3.3, we have $\left.\widehat{\Psi}_{\text {diff }}^{\text {joint }}(x, y)=\sum_{z \in \mathbb{Z}} \widehat{\Psi}^{\text {joint }}((x, 0),(z+y, z))\right)$.

Because $X^{z_{1}}$ and $X^{z_{2}}$ have bounded increments, (2.6) implies an exponential tail bound for jump sizes under $\widehat{\Psi}_{\text {diff }}^{\text {joint }}$ :

$$
\widehat{\Psi}_{\text {diff }}^{\text {joint }}(x, y) \leqslant C e^{-c|y-x|} \text { for } x, y \in \mathbb{Z} \text {. }
$$

One can implement the same construction when the two walks $X^{z_{1}}$ and $X^{z_{2}}$ move independently on independent copies of the oriented percolation cluster (formally, let $\omega^{\prime}$ be an independent copy of $\omega$ and $\widetilde{\omega}^{\prime}$ an independent copy of $\widetilde{\omega}$, then construct $X^{z_{2}}$ by using $\omega^{\prime}$ and $\widetilde{\omega}^{\prime}$ in (1.3) and (1.4); we condition now on $z_{1} \rightarrow^{\omega} \infty$ and $\left.z_{2} \rightarrow \omega^{\prime} \infty\right)$. Then $\left(\widehat{D}_{\ell}^{z_{1}, z_{2}}\right)_{\ell}$ is again a Markov chain on $\mathbb{Z}$, we denote its transition probability matrix in this case by $\widehat{\Psi}_{\text {diff }}^{\text {ind }}$. In fact, $\widehat{\Psi}_{\text {diff }}^{\text {ind }}$ is irreducible, symmetric and spatially homogeneous (i.e., $\widehat{D}^{z_{1}, z_{2}}$ is now a symmetric random walk) with exponentially bounded tails

$$
\widehat{\Psi}_{\text {diff }}^{\text {ind }}(x, y)=\widehat{\Psi}_{\text {diff }}^{\text {ind }}(y, x)=\widehat{\Psi}_{\text {diff }}^{\text {ind }}(0, y-x) \leqslant C e^{-c|y-x|} \quad \text { for } x, y \in \mathbb{Z},
$$

see Birkner et al. (2013), Section 3.1, especially the discussion after Remark 3.3.

Using a coupling construction and space-time mixing properties of the percolation cluster, one finds the following lemma.

Lemma 2.2 (Birkner et al. (2013, Lemma 3.4)). We have $\left(\|\cdot\|_{\mathrm{TV}}\right.$ denotes total variation distance)

$$
\left\|\widehat{\Psi}_{\mathrm{diff}}^{\mathrm{joint}}(x, \cdot)-\widehat{\Psi}_{\mathrm{diff}}^{\mathrm{ind}}(x, \cdot)\right\|_{\mathrm{TV}} \leqslant C e^{-c|x|} \quad \text { for all } x \in \mathbb{Z} .
$$


Remark 2.3. One can in complete analogy to the construction for $m=2$ walks consider joint regeneration times for any number $m \geqslant 2$ of walks $X^{\left(x_{1}, n_{1}\right)}, \ldots, X^{\left(x_{m}, n_{m}\right)}$ (obviously, joint regeneration can then only occur after "real" time $\max \left\{n_{1}, n_{2}, \ldots\right.$, $\left.n_{m}\right\}$ ). In fact, in Section 2.3.3 we will consider the case $m=5$.

Following the construction in Birkner et al. (2013, Section 3), one obtains that a tail bound for increments between joint regeneration times analogous to (2.6) also holds in this case.

2.2. A bound on the meeting time for two walks on the cluster. Let $T_{\text {meet }}^{\left(z_{1}, z_{2}\right)}:=$ $\inf \left\{n \geqslant 0: X_{n}^{\left(z_{1}\right)}=X_{n}^{\left(z_{2}\right)}\right\}$ (with the usual convention inf $\varnothing=+\infty$ ).

Lemma 2.4. There is $C=C(p)<\infty$ such that

$$
\widetilde{\mathbb{P}}_{z_{1}, z_{2}}\left(T_{\text {meet }}^{\left(z_{1}, z_{2}\right)}>n\right) \leqslant C \frac{\left|z_{1}-z_{2}\right|}{\sqrt{n}} \text { for } z_{1}, z_{2} \in \mathbb{Z}, n \in \mathbb{N} .
$$

In particular, $\widetilde{\mathbb{P}}_{z_{1}, z_{2}}\left(T_{\text {meet }}^{\left(z_{1}, z_{2}\right)}<\infty\right)=1$ and hence also $P_{\omega}\left(T_{\text {meet }}^{\left(z_{1}, z_{2}\right)}<\infty\right)=1$ for $\mathbb{P}$-almost all $\omega \in B_{\left(z_{1}, 0\right)} \cap B_{\left(z_{2}, 0\right)}$.

Instead of conditioning on $\left(z_{1}, 0\right),\left(z_{2}, 0\right) \in \mathcal{C}$ in $(2.11)$, we could also pick the "nearest" connected sites (say, on the left, as in (1.8)) without changing the statement.

We are interested in collision events of two directed random walks $X^{\left(z_{1}\right)}, X^{\left(z_{2}\right)}$ moving on the same space-time cluster $\mathcal{C}$, i.e., we ask that the two walks are at the same time at the same site. Lemma 2.4 tells us in particular that a collision event between two random walks occurs almost surely in dimension $d=1$. This is not completely obvious a priori because "holes" in the space-time cluster $\mathcal{C}$ might at least in principle prevent such collisions. However, the right-hand side of (2.11) is - modulo a constant - also the correct order for the corresponding probability for two simple random walks on $\mathbb{Z}$, so that in this sense, the holes in the cluster do not have a strong influence.

Fix $z_{1}, z_{2}$, put

$$
\widehat{T}_{\text {meet }}^{\left(z_{1}, z_{2}\right)}:=\inf \left\{\ell \in \mathbb{N}: \hat{X}_{\ell}^{z_{1}}=\hat{X}_{\ell}^{z_{2}}\right\}=\inf \left\{\ell \in \mathbb{N}: \widehat{D}_{\ell}^{z_{1}, z_{2}}=0\right\} .
$$

In view of (2.6), it suffices to establish that there is a constant $C=C(p)<\infty$ such that

$$
\widetilde{\mathbb{P}}_{z_{1}, z_{2}}\left(\widehat{T}_{\text {meet }}^{\left(z_{1}, z_{2}\right)}>n\right) \leqslant C \frac{\left|z_{1}-z_{2}\right|}{\sqrt{n}} \text { for } z_{1}, z_{2} \in \mathbb{Z}, n \in \mathbb{N} .
$$

(To pass from $\widehat{T}_{\text {meet }}^{\left(z_{1}, z_{2}\right)}$ to $T_{\text {meet }}^{\left(z_{1}, z_{2}\right)}$ note that if $k(n)$ denotes the last simultaneous regeneration time before time $n$, for sufficiently small $c>0$, the probability of the event $\{k(n) / n<c\}$ decays exponentially as $n \rightarrow \infty$.)

The key ingredient for the proof of Lemma 2.4 is the estimate on the total variation error between $\widehat{\Psi}_{\text {diff }}^{\text {joint }}$ and $\widehat{\Psi}_{\text {diff }}^{\text {ind }}$ recalled in Lemma 2.2. Lemma 2.4 is thus in a sense a "trivial" instance of a so-called Lamperti problem, $\left(\hat{D}_{\ell}^{z_{1}, z_{2}}\right)_{\ell}$ is under $\widetilde{\mathbb{P}}_{z_{1}, z_{2}}$ a Markov chain that is a local perturbation of a symmetric random walk and the drift at $x$ vanishes exponentially fast in $|x|$. A very fine analysis in the case of \pm 1 -steps can be found in Alexander (2011), see also the references there for background. Denisov et al. (2016) have established a generalization of Alexander's results to the non-nearest neighbour case which in particular refines 
(2.11) to asymptotic equivalence as $n \rightarrow \infty$ (see Denisov et al. (2016) Thm. 5.11 and Lemma 5.12; cf also Cor. 5.16 for the hitting time of a point instead of a half-interval). A recent and equally enjoyable reference on Lamperti problems is Menshikov et al. (2017). For completeness' sake we present here a short, rough proof of the coarser estimate that suffices for our purposes. (More detailed arguments can also be found in Steiber (2017, Chapter 2).)

(Sketchy) proof of Lemma 2.4: Write $\left(\widehat{D}_{n}\right)_{n \in \mathbb{N}_{0}}$ for the Markov chain on $\mathbb{Z}$ with transition probabilities $\widehat{\Psi}_{\text {diff }}^{\text {joint }}$. For $x \in \mathbb{Z}$ we will write here $\mathbb{P}_{x}^{\text {joint }}$ for a probability measure under which this Markov chain starts in $x$, i.e., $\mathbb{P}_{x}^{j o i n t}\left(\widehat{D}_{0}=x\right)=1$.

Let us first verify that there exists $x_{0}>0, n_{0}>0$ and $C$ such that

$$
\mathbb{P}_{x}^{\text {joint }}\left(\tau_{x_{0}}>n\right) \leqslant C \frac{|x|}{\sqrt{n}} \text { for all } n \geqslant n_{0}, x \in \mathbb{Z}
$$

where

$$
\tau_{x_{0}}:=\inf \left\{n \geqslant 0:\left|\widehat{D}_{n}\right| \leqslant x_{0}\right\} .
$$

By Lemma 2.2 and analogous properties of $\widehat{\Psi}_{\text {diff }}^{\text {ind }}$ we have

$$
\left|\mathbb{E}\left[\widehat{D}_{n+1}-x \mid \widehat{D}_{n}=x\right]\right| \leqslant C e^{-c x} \quad \text { and } \operatorname{Var}\left[\widehat{D}_{n+1} \mid \widehat{D}_{n}=x\right] \geqslant \tilde{\sigma}^{2}
$$

whenever $|x|$ is sufficiently large (for suitable $\tilde{\sigma}^{2}, c, C \in(0, \infty)$ ).

We can find $c_{1}, x_{0} \in(0, \infty)$ such that the function

$$
f(x)=\int_{0}^{|x|} \exp \left(2 e^{-c_{1} y} / c_{1}\right) d y, \quad x \in \mathbb{R}
$$

is non-negative and superharmonic for $\widehat{\Psi}_{\text {diff }}^{\text {joint }}$ in $\mathbb{Z} \cap\left[-x_{0}, x_{0}\right]^{c}$. This follows from Lemma 2.2 and a Taylor expansion of $f$ to second order (more details are given in Appendix A). Note that $f$ solves $\frac{1}{2} f^{\prime \prime}(x)+\operatorname{sgn}(x) e^{-c_{1}|x|} f^{\prime}(x)=0$ for $x \neq 0$, i.e., $f$ is a harmonic function for a Brownian motion with spatially inhomogeneous drift $\operatorname{sgn}(x) e^{-c_{1}|x|}$. Note that $f(x)$ can in principle be expressed explicitly in terms of the exponential integral function (see, e.g., Abramowitz and Stegun (1964, Chapter 5)), for our purposes it suffices to observe that $0 \leqslant f(x) \leqslant e^{2 / c_{1}}|x|$.

Thus, starting from $\widehat{D}_{0}=x$ with $|x|>x_{0}, Z_{n}:=f\left(\widehat{D}_{n \wedge \tau_{x_{0}}}\right)$ is a non-negative supermartingale with $Z_{0}=f(x) \leqslant c|x|$ and it is easy to see (cf (2.16)) that for some $b_{1}<\infty, 0<b_{2}<\infty$

$\left|\mathbb{E}\left[Z_{n+1} \mid \sigma\left(Z_{0}, \ldots, Z_{n}\right)\right]-Z_{n}\right| \leqslant b_{1}, \quad \operatorname{Var}\left[Z_{n+1} \mid \sigma\left(Z_{0}, \ldots, Z_{n}\right)\right] \geqslant b_{2}$ on $\left\{\tau_{x_{0}}>n\right\}$

(2.14) follows then from well known tail bounds for hitting times of supermartingales (see, e.g., Levin et al. (2009, Proposition 17.20)).

Obtaining (2.13) from (2.14) is a fairly standard argument for irreducible Markov chains: We can find $M<\infty, \varepsilon>0$ such that

$\inf _{|x| \leqslant x_{0}} \mathbb{P}_{x}^{\text {joint }}\left(\widehat{D}\right.$ hits 0 within at most $M$ steps without exiting $\left[-x_{0}, x_{0}\right]$ before $) \geqslant \varepsilon$. 
Thus, starting from some $x \in\left[-x_{0}, x_{0}\right]$, the path of $\widehat{D}$ before hitting 0 can be decomposed into an at most geometrically distributed number of "outside excursions" out of $\left[-x_{0}, x_{0}\right]$ and path pieces inside $\left[-x_{0}, x_{0}\right]$, plus the final piece inside $\left[-x_{0}, x_{0}\right]$ when 0 is hit for the first time. By (2.14) and the (exponential) tail bounds on jumps sizes for $\widehat{\Psi}_{\text {diff }}^{\text {joint }}$, the tail of the length distribution of an outside excursion is bounded by $C / \sqrt{n}$ (uniformly in $n \geqslant n_{0}$ and the starting point inside), the length distribution of the pieces "inside" has (again uniformly in the starting point inside) exponentially decaying tails. It is well known that a geometric sum of non-negative random variables with a tail bound of the form $C / \sqrt{n}$ again satisfies such a tail bound (with an enlarged $C$ ), thus there are $C<\infty$ and $n_{0} \in \mathbb{N}$ with

$$
\sup _{|x| \leqslant x_{0}} \mathbb{P}_{x}^{\text {joint }}\left(\inf \left\{\ell \geqslant 0: \hat{D}_{\ell}=0\right\}>n\right) \leqslant \frac{C}{\sqrt{n}} \text { for all } n \geqslant n_{0},
$$

see, e.g. the proof of Corollary 5.16 in Denisov et al. (2016).

Now (2.13), with a suitably enlarged $C$, is for $\left|z_{1}-z_{2}\right| \leqslant x_{0}$ immediate from (2.20), for $\left|z_{1}-z_{2}\right|>x_{0}$ it follows from (2.14) and (2.20) since

$\widetilde{\mathbb{P}}_{z_{1}, z_{2}}\left(\widehat{T}_{\text {meet }}^{\left(z_{1}, z_{2}\right)}>n\right) \leqslant \mathbb{P}_{z_{1}-z_{2}}^{\text {joint }}\left(\tau_{x_{0}}>n / 2\right)+\sup _{|x|<x_{0}} \mathbb{P}_{x}^{\text {joint }}\left(\inf \left\{\ell \geqslant 0: \hat{D}_{\ell}=0\right\}>n / 2\right)$.

Remark 2.5. Put $\sigma_{y}:=\inf \left\{n \geqslant 0:\left|\widehat{D}_{n}\right| \geqslant y\right\}$ (and recall $\tau_{x_{0}}$ from (2.15) and $x_{0}$ from the proof of Lemma 2.4). We see from the proof of Lemma 2.4 that there exist $y_{0} \in \mathbb{N}$ and $c<\infty$ so that

$$
\mathbb{P}_{x}^{\text {joint }}\left(\tau_{x_{0}}>\sigma_{y}\right) \leqslant c \frac{x}{y} \text { for all } 2 x_{0}<x<y \text { and } y \geqslant y_{0} .
$$

Proof: With $f$ from (2.17) and $\tau:=\tau_{x_{0}} \wedge \sigma_{y}$, the process $\left(f\left(\widehat{D}_{n \wedge \tau}\right)\right)_{n \in \mathbb{N}_{0}}$ is a nonnegative supermartingale (w.r.t. the filtration generated by the Markov chain $\widehat{D}$ ), thus by optional stopping

$$
\begin{aligned}
f(x) \geqslant & \mathbb{E}_{x}^{\text {joint }}\left[f\left(\widehat{D}_{\tau}\right)\right] \\
= & \mathbb{P}_{x}^{\text {joint }}\left(\tau_{x_{0}}>\sigma_{y}\right) \mathbb{E}_{x}^{\text {joint }}\left[f\left(\widehat{D}_{\tau}\right) \mid \tau_{x_{0}}>\sigma_{y}\right] \\
& \quad+\left(1-\mathbb{P}_{x}^{\text {joint }}\left(\tau_{x_{0}}>\sigma_{y}\right)\right) \mathbb{E}_{x}^{\text {joint }}\left[f\left(\widehat{D}_{\tau}\right) \mid \tau_{x_{0}}<\sigma_{y}\right] \\
\geqslant & \mathbb{P}_{x}^{\text {joint }}\left(\tau_{x_{0}}>\sigma_{y}\right)\left(f(y)-f\left(x_{0}\right)\right)+f\left(x_{0}\right) .
\end{aligned}
$$

This together with $|x| \leqslant f(x) \leqslant e^{2 / c_{1}}|x|$ implies (2.21).

The following lemma allows to control the undesirable situation that two walks come close but then separate again and spend a long time apart before eventually coalescing. We will need this in Section 2.3.2 below (Checking condition $\left(I_{1}\right)$, Step 2).

Lemma 2.6. For $z_{1}=\left(x_{1}, t_{1}\right), z_{2}=\left(x_{2}, t_{2}\right) \in \mathbb{Z} \times \mathbb{Z}$ write

$$
\begin{aligned}
& T_{\text {near }}^{z_{1}, z_{2}}:=\inf \left\{t \in \mathbb{Z}, t \geqslant t_{1} \vee t_{2}:\left|\pi^{z_{1}}(t)-\pi^{z_{2}}(t)\right| \leqslant 1\right\}, \\
& T_{\text {meet }}^{z_{1}, z_{2}}:=\inf \left\{t \in \mathbb{Z}, t \geqslant t_{1} \vee t_{2}: \pi^{z_{1}}(t)=\pi^{z_{2}}(t)\right\} \quad\left(\geqslant T_{\text {near }}^{z_{1}, z_{2}}\right) .
\end{aligned}
$$

The family $\left\{T_{\text {meet }}^{z_{1}, z_{2}}-T_{\text {near }}^{z_{1}, z_{2}}: z_{1}, z_{2} \in \mathbb{Z} \times \mathbb{Z}\right\}$ is tight, that is

$$
\lim _{M \rightarrow \infty} \sup _{z_{1}, z_{2} \in \mathbb{Z} \times \mathbb{Z}} \mathbb{P}\left(T_{\text {meet }}^{z_{1}, z_{2}}-T_{\text {near }}^{z_{1}, z_{2}} \geqslant M\right)=0 .
$$


In particular

$$
\lim _{M \rightarrow \infty} \sup _{z_{1}, z_{2} \in \mathbb{Z} \times \mathbb{Z}} \mathbb{P}\left(\sup \left\{\left|\pi^{z_{1}}(t)-\pi^{z_{2}}(t)\right|: T_{\text {near }}^{z_{1}, z_{2}} \leqslant t \leqslant T_{\text {meet }}^{z_{1}, z_{2}}\right\} \geqslant M\right)=0 .
$$

Proof sketch: (2.26) follows from (2.25) because $\left|\pi^{z_{1}}\left(T_{\text {near }}^{z_{1}, z_{2}}\right)-\pi^{z_{2}}\left(T_{\text {near }}^{z_{1}, z_{2}}\right)\right| \leqslant 1$ and thus

$$
\sup \left\{\left|\pi^{z_{1}}(t)-\pi^{z_{2}}(t)\right|: T_{\text {near }}^{z_{1}, z_{2}} \leqslant t \leqslant T_{\text {meet }}^{z_{1}, z_{2}}\right\} \leqslant 1+2\left(T_{\text {meet }}^{z_{1}, z_{2}}-T_{\text {near }}^{z_{1}, z_{2}}\right) .
$$

For (2.25), consider first the case $t_{1}=t_{2}$, and then w.l.o.g. $t_{1}=t_{2}=0, x_{1}=0$. Write

$$
X_{n}=\pi^{z_{1}}(n), \quad X_{n}^{\prime}=\pi^{z_{2}}(n), \quad n \in \mathbb{N}_{0}
$$

for the two walks. The idea behind (2.25) is that even if $\left|\pi^{z_{1}}\left(T_{\text {near }}^{z_{1}, z_{2}}\right)-\pi^{z_{2}}\left(T_{\text {near }}^{z_{1}, z_{2}}\right)\right|=$ 1 and thus $T_{\text {near }}^{z_{1}, z_{2}}<T_{\text {meet }}^{z_{1}, z_{2}}$, the difference should be bounded in probability irrespective of where the two walks are at time $T_{\text {near }}^{z_{1}, z_{2}}$ in view of Lemma 2.4. A little complication lies in the fact that the pair $\left(X_{n}, X_{n}^{\prime}\right)_{n}$ is not in itself a Markov chain, so we cannot simply stop at the random time $T_{\text {near }}^{z_{1} z_{2}}$ and then apply the strong Markov property.

Instead, we consider the two walks along their joint regeneration times $0=$ $T_{0}^{\operatorname{sim}}<T_{1}^{\operatorname{sim}}<T_{2}^{\text {sim }}<\cdots$, which yields a Markov chain $\left(\hat{X}_{\ell}, \hat{X}_{\ell}^{\prime}\right)_{\ell \in \mathbb{N}_{0}}$ (recall the discussion and notation from Section 2.1). For $a>0$ put

$$
\widehat{T}(a):=\inf \left\{\ell \in \mathbb{N}_{0}:\left|\widehat{X}_{\ell}-\hat{X}_{\ell}^{\prime}\right| \leqslant a\right\} .
$$

Fix $M>0$ and let $k \in \mathbb{N}$ be the smallest integer such that $2^{k} M \geqslant\left|x_{2}-x_{1}\right|$. Then

$$
\begin{aligned}
& \mathbb{P}\left(\left|X_{n}-X_{n}^{\prime}\right| \leqslant 1 \text { for some } n<T_{\widehat{T}(M)}^{\operatorname{sim}}\right) \\
& \leqslant \sum_{j=1}^{k} \mathbb{P}\left(\left|X_{n}-X_{n}^{\prime}\right| \leqslant 1 \text { for some } T_{\widehat{T}\left(2^{j} M\right)}^{\operatorname{sim}} \leqslant n<T_{\widehat{T}\left(2^{j-1} M\right)}^{\operatorname{sim}}\right) \\
& \leqslant \sum_{j=1}^{k} \mathbb{P}\left(T_{\ell}^{\text {sim }}-T_{\ell-1}^{\text {sim }}>2^{j-2} M \text { for some } \widehat{T}\left(2^{j} M\right) \leqslant \ell<\widehat{T}\left(2^{j-1} M\right)\right) \\
& \leqslant \sum_{j=1}^{k}\left\{\mathbb{P}\left(\widehat{T}\left(2^{j-1} M\right)-\widehat{T}\left(2^{j} M\right)>\left(2^{j} M\right)^{3}\right)\right. \\
& \quad+\mathbb{P}\left(T_{\ell}^{\operatorname{sim}}-T_{\ell-1}^{\operatorname{sim}}>2^{j-2} M \text { for some } \ell\right. \\
& \left.\left.\quad \text { with } \widehat{T}\left(2^{j} M\right) \leqslant \ell \leqslant \widehat{T}\left(2^{j} M\right)+\left(2^{j} M\right)^{3}\right)\right\} \\
& \leqslant \\
& \sum_{j=1}^{k}\left\{\frac{C M 2^{j}}{\sqrt{\left(2^{j} M\right)^{3}}}+\left(2^{j} M\right)^{3} C \exp \left(-c 2^{j-2} M\right)\right\} \\
& \leqslant \frac{C}{M^{1 / 2}} \sum_{j=1}^{\infty} \frac{1}{2^{j / 2}}+C M^{3} \sum_{j=1}^{\infty} \exp \left(j \log (2)-c 2^{j-1} M\right)=: b(M) .
\end{aligned}
$$

Note that $b(M) \rightarrow 0$ as $M \rightarrow \infty$.

We can apply the Markov property of $\left(\hat{X}_{\ell}, \hat{X}_{\ell}^{\prime}\right)$ at the stopping time $\widehat{T}(M)$ (which corresponds to time $T_{\hat{T}(M)}^{\mathrm{sim}}$ for the two walks $\left(X_{n}, X_{n}^{\prime}\right)$ themselves), noting that

$$
\left|X_{T_{\widehat{T}(M)}^{\operatorname{sim}}}-X_{T_{\widehat{T}(M)}^{\operatorname{sim}}}^{\prime}\right|=\left|\hat{X}_{\widehat{T}(M)}-\hat{X}_{\widehat{T}(M)}^{\prime}\right| \leqslant M
$$


and thus, using shift-invariance of the joint distribution and Lemma 2.4,

$$
\begin{aligned}
& \mathbb{P}\left(T_{\text {meet }}^{z_{1}, z_{2}}-T_{\text {near }}^{z_{1}, z_{2}}>M^{5}\right) \\
& \leqslant \mathbb{P}\left(\left|X_{n}-X_{n}^{\prime}\right| \leqslant 1 \text { for some } n \leqslant T_{\widehat{T}(M)}^{\text {sim }}\right)+\sum_{x=1}^{M} \widetilde{\mathbb{P}}_{0, x}\left(T_{\text {meet }}^{(0, x)}>M^{5}\right) \\
& \leqslant b(M)+C \sum_{x=1}^{M} \frac{x}{M^{5 / 2}} \underset{M \rightarrow \infty}{\longrightarrow} 0
\end{aligned}
$$

and the bound in the last line holds uniformly for all $z_{1}, z_{2} \in \mathbb{Z} \times \mathbb{Z}$.

When $t_{1} \neq t_{2}$, say $t_{2}>t_{1}$, we let the first walk begin at time $t_{1}$ and "run freely" until time $t_{2}$, then argue as above. Again, there is a slight complication because we would have to first look only along regeneration times, then use joint regeneration times as soon as the second walk "comes into the picture". This can be handled similarly as above, we do not spell out the details.

2.3. Proof of Theorem 1.1. We follow the approach developed in Newman et al. (2005) and Sun (2005).

2.3.1. Conditions for convergence to the Brownian web. First we introduce a little more notation which is needed to formulate the sufficient conditions for convergence to the Brownian web from Newman et al. (2005). Define $\Lambda_{L, T}:=[-L, L] \times$ $[-T, T] \subset \mathbb{R}^{2}$. For $x_{0}, t_{0} \in \mathbb{R}$ and $u, t>0$ let $R\left(x_{0}, t_{0}, u, t\right)$ be the rectangle $\left[x_{0}-u, x_{0}+u\right] \times\left[t_{0}, t_{0}+t\right] \subset \mathbb{R}^{2}$ and define $A_{t, u}\left(x_{0}, t_{0}\right)$ to be the set of $K \in \mathcal{H}$ which contain a path that touches both, the rectangle $R\left(x_{0}, t_{0}, u, t\right)$ and the left or right boundary of the bigger rectangle $R\left(x_{0}, t_{0}, 20 u, 2 t\right)$ (note $A_{t, u}\left(x_{0}, t_{0}\right) \in \mathcal{B}_{\mathcal{H}}$ ). For $a, b, t_{0}, t \in \mathbb{R}, a<b, t>0$ and $K \in \mathcal{H}$, we define the number of distinct points in $\mathbb{R} \times\left\{t_{0}+t\right\}$, which are touched by some path in $K$ that also touches $[a, b] \times\left\{t_{0}\right\}$ by

$$
\begin{aligned}
\eta\left(t_{0}, t ; a, b\right): & =\eta_{K}\left(t_{0}, t ; a, b\right) \\
& :=\#\left\{y \in \mathbb{R}: \begin{array}{rl}
\exists & \\
& \text { touches both } \left.\left(x, t_{0}\right) \text { and }\left(y, t_{0}+t\right)\right\} .
\end{array}\right.
\end{aligned}
$$

Similarly, let

$$
\begin{aligned}
& \widehat{\eta}\left(t_{0}, t ; a, b\right):=\widehat{\eta}_{K}\left(t_{0}, t ; a, b\right) \\
& :=\#\left\{x \in(a, b) \text { there is a path in } K \text { which touches both } \mathbb{R} \times\left\{t_{0}\right\} \text { and }\left(x, t_{0}+t\right)\right\} .
\end{aligned}
$$

be the number of points in $(a, b) \times\left\{t+t_{0}\right\}$ which are touched by some path in $K$ which started at time $t_{0}$ or before.

If $\mathcal{X}$ is a $\left(\mathcal{H}, \mathcal{B}_{\mathcal{H}}\right)$-valued random variable, we define

$\mathcal{X}^{s^{-}}$to be the subset of paths in $\mathcal{X}$ which start before or at time $s$.

Combining Theorem 1.4 and Lemma 6.1 from Newman et al. (2005), we see that a family $\left\{\mathcal{X}_{n}\right\}_{n}$ of $\left(\mathcal{H}, \mathcal{B}_{\mathcal{H}}\right)$-valued random variables with distribution $\left\{\mu_{n}\right\}_{n}$ converges in distribution to the standard Brownian web $\mathcal{W}$, if it satisfies the following conditions: 
$\left(I_{1}\right)$ There exist single path valued random variables $\theta_{n}^{y} \in \mathcal{X}_{n}$, for $y \in \mathbb{R}^{2}$, satisfying:

for $\mathcal{D}$ a deterministic countable dense subset of $\mathbb{R}^{2}$, for any deterministic $z_{1}, \ldots, z_{m} \in \mathcal{D}, \theta_{n}^{z_{1}}, \ldots, \theta_{n}^{z_{m}}$ converge jointly in distribution as $n \rightarrow \infty$ to coalescing Brownian motions (with unit diffusion constant) starting at $z_{1}, \ldots, z_{m}$

$\left(T_{1}\right)$ For every $u, L, T \in(0, \infty)$

$$
\widetilde{g}(t, u ; L, T) \equiv t^{-1} \limsup _{n \rightarrow \infty} \sup _{\left(x_{0}, t_{0}\right) \in \Lambda_{L, T}} \mu_{n}\left(A_{t, u}\left(x_{0}, t_{0}\right)\right) \longrightarrow 0 \text { as } t \rightarrow 0^{+},
$$

which is a sufficient condition for the family $\left\{\mathcal{X}_{n}\right\}_{n}$ to be tight.

$\left(B_{1}^{\prime}\right)$ For all $\beta>0$

$$
\limsup _{n \rightarrow \infty} \sup _{t>\beta} \sup _{t_{0}, a \in \mathbb{R}} \mu_{n}\left(\eta\left(t_{0}, t ; a-\varepsilon, a+\varepsilon\right)>1\right) \longrightarrow 0 \text { as } \varepsilon \rightarrow 0^{+} .
$$

$\left(E_{1}^{\prime}\right)$ If $\mathcal{Z}_{t_{0}}$ is any subsequential limit of $\left\{\mathcal{X}_{n}^{t_{0}^{-}}\right\}_{n}$ for any $t_{0} \in \mathbb{R}$, then for all $t, a, b \in \mathbb{R}$, with $t>0$ and $a<b$,

$$
\mathbb{E}\left[\widehat{\eta}_{\mathcal{Z}_{t_{0}}}\left(t_{0}, t ; a, b\right)\right] \leqslant \mathbb{E}\left[\widehat{\eta}_{\mathcal{W}}\left(t_{0}, t ; a, b\right)\right]=\frac{b-a}{\sqrt{\pi t}} .
$$

Remark 2.7. 1. We consider the diffusively rescaled closure of $\boldsymbol{\Gamma}=\left\{\pi^{z}: z \in \mathbb{Z} \times \mathbb{Z}\right\}=$ $\left\{\pi^{z}: z \in \mathcal{C}\right\}$, which is the collection of all linearly interpolated random walk paths. Therefore, instead of $\mathcal{X}_{n}$, we usually write $\mathcal{X}_{\delta}$ to denote the $\left(\mathcal{H}, \mathcal{B}_{\mathcal{H}}\right)$-valued random variable $S_{v, \delta} \boldsymbol{\Gamma}$. If we want to consider the weak limit of $\left(\mathcal{X}_{\delta}\right)_{\delta>0}$ along a certain subsequence $\left(\delta_{n}\right)_{n}$, where $\delta_{n} \rightarrow 0$ as $n \rightarrow \infty$, we denote the random variables $S_{v, \delta_{n}} \boldsymbol{\Gamma}$ by $\mathcal{X}_{\delta_{n}}$. The probability measure $\mathbb{P} \circ\left(S_{v, \delta_{n}} \boldsymbol{\Gamma}\right)^{-1}$ on $\left(\mathcal{H}, \mathcal{B}_{\mathcal{H}}\right)$ is denoted by $\mu_{\delta_{n}}$.

2. We invoke condition $\left(E_{1}^{\prime}\right)$ because because in our model, paths $\pi^{z_{1}}$ and $\pi^{z_{2}}$ can cross each other without coalescing. In this respect, our scenario is different from that in Sarkar and Sun (2013).

2.3.2. Checking condition $\left(I_{1}\right)$. Let $\mathcal{D}$ be a dense countable subset of $\mathbb{R}^{2}$ and choose distinct $y_{1}=\left(x_{1}, t_{1}\right), \ldots, y_{m}=\left(x_{m}, t_{m}\right) \in \mathcal{D}$. Define $y_{\delta, i}:=\left(\left\lfloor x_{i} v \delta^{-1}\right\rfloor,\left\lfloor t_{i} \delta^{-2}\right\rfloor\right)$. Let

$$
\pi_{\delta}^{i}:=S_{v, \delta}^{1} \circ \pi^{y_{\delta, i}}, \quad i=1, \ldots, m
$$

be the corresponding diffusively rescaled (and coalescing) random walks. In order to show that $\left(\pi_{\delta}^{1}, \ldots, \pi_{\delta}^{m}\right)$ converges to a system of $m$ coalescing Brownian motions as $\delta \rightarrow 0$, we will follow the strategy from Newman et al. (2005) and construct a suitable coupling with $m$ independent walks on the cluster. One could alternatively attempt to use the characterization of coalescing Brownian motions via a martingale problem, we discuss this briefly in Remark 2.8 below.

We will need some auxiliary types of paths: Let $\tilde{X}^{\left(c\left(y_{\delta, i}\right)\right)}=\left(\tilde{X}_{t}^{\left(c\left(y_{\delta, i}\right)\right)}\right)_{t \geqslant\left\lfloor t_{i} / \delta^{2}\right\rfloor}$ be independent conditionally on $\mathcal{C}$ with transition probabilities given by (1.5), i.e., $\tilde{X}^{\left(c\left(y_{\delta, i}\right)\right)}, \ldots, \tilde{X}^{\left(c\left(y_{\delta, m}\right)\right)}$ are $m$ independent walks on the same realization of the cluster, with $\tilde{X}^{\left(c\left(y_{\delta, i}\right)\right)}$ starting from the nearest possible starting point to $y_{\delta, i}$ on $\mathcal{C}$ (recall $c(z)$ from (1.8)). Note that we can for example construct these walks as in (1.4) and (1.3) by using $m$ independent copies of $\widetilde{\omega}$. Let $\widetilde{\pi}^{\left(c\left(y_{\delta, i}\right)\right)}$ be the extension of $\widetilde{X}^{\left(c\left(y_{\delta, i}\right)\right)}$ to real times by linear interpolation, and denote their rescalings by

$$
\tilde{\pi}_{\delta}^{i}:=S_{v, \delta}^{1} \circ \tilde{\pi}^{\left(c\left(y_{\delta, i}\right)\right)}, \quad i=1, \ldots, m .
$$


Note that $\widetilde{\pi}_{\delta}^{i}={ }^{d} \pi_{\delta}^{i}$ for every $i$ but unlike the $\pi_{\delta}^{i}$ 's, different paths $\widetilde{\pi}_{\delta}^{i}$ and $\widetilde{\pi}_{\delta}^{j}$ with $j \neq i$ can meet at times $\in \delta^{2} \mathbb{Z}$ and then separate again.

Furthermore, we need two different coalescence rules on $\Pi^{m}$ : Under the first rule $\Gamma_{\alpha}$, paths are merged when they first coincide. Let $\left(\left(f_{1}, \sigma_{1}\right), \ldots,\left(f_{m}, \sigma_{m}\right)\right) \in \Pi^{m}$. Define

$$
T_{\alpha}^{i, j}:=\inf \left\{t>\sigma_{i} \vee \sigma_{j}, t \in \mathbb{R}: f_{i}(t)=f_{j}(t)\right\} .
$$

Note that $t \in \mathbb{R}$ can be arbitrary, in particular $t \notin \delta^{2} \mathbb{Z}$ is possible.

Start with the (trivial) equivalence relation $i \sim i, i \nsim j$ for all $i \neq j$ on $\{1, \ldots, m\}$. Define

and

$$
\tau_{\alpha}:=\min _{1 \leqslant i, j \leqslant m, i \rtimes j} T_{\alpha}^{i, j}, \quad \text { with } \min \varnothing=\infty
$$

$$
\Gamma_{\alpha}\left(f_{i}(t)\right):= \begin{cases}f_{i}(t), & \text { if } t<\tau_{\alpha} \\ f_{i *}(t), & \text { if } t \geqslant \tau_{\alpha}\end{cases}
$$

where $i^{*}=\min \left\{j:(j \sim i)\right.$ or $\left(j \nsim i\right.$ and $\left.\left.T_{\alpha}^{i, j}=\tau_{\alpha}\right)\right\}$. Update the equivalence relation at time $\tau_{\alpha}$ by assigning $i \sim i^{*}$ (and implicitly also $i \sim i^{\prime}$ for all $i^{\prime} \sim i^{*}$ ). Iterating this procedure, we get the desired structure of coalescing random walks. We label the successive times $\tau_{\alpha}$ by $\tau_{\alpha}^{1}, \ldots, \tau_{\alpha}^{k}$, where $k \in\{1, \ldots, m\}$ is the smallest index such that $\tau_{\alpha}^{k}=\infty$ (after $k$ steps, either all paths have been merged or no further meeting of paths occurs). We will denote the resulting $m$-tuple of paths by $\Gamma_{\alpha}\left(\left(f_{1}, \sigma_{1}\right), \ldots,\left(f_{m}, \sigma_{m}\right)\right)$.

When we apply $\Gamma_{\alpha}$ to $\left(\pi_{\delta}^{1}, \ldots, \pi_{\delta}^{m}\right)$ it may because of the linear interpolation happen that paths are merged even though the underlying discrete walks did not meet. This is not literally the correct dynamics and is not the case for the second coalescence rule $\Gamma_{\beta, \delta}$.

$\Gamma_{\beta, \delta}: \Pi^{m} \rightarrow \Pi^{m}$ is defined analogously to $\Gamma_{\alpha}$ except that we replace in the construction $T_{\alpha}^{i, j}$ by

$$
T_{\beta, \delta}^{i, j}:=\inf \left\{t \in \delta^{2} \mathbb{Z}: t \geqslant \sigma_{i} \vee \sigma_{j} \text { and } f_{i}(t)=f_{j}(t)\right\} .
$$

Note that by construction

$$
\left(\Gamma_{\alpha}\left(\tilde{\pi}_{\delta}^{1}, \ldots, \tilde{\pi}_{\delta}^{m}\right), \Gamma_{\beta, \delta}\left(\tilde{\pi}_{\delta}^{1}, \ldots, \tilde{\pi}_{\delta}^{m}\right)\right) \stackrel{d}{=}\left(\Gamma_{\alpha}\left(\pi_{\delta}^{1}, \ldots, \pi_{\delta}^{m}\right),\left(\pi_{\delta}^{1}, \ldots, \pi_{\delta}^{m}\right)\right),
$$

thus in particular

$$
\Gamma_{\beta, \delta}\left(\tilde{\pi}_{\delta}^{1}, \ldots, \tilde{\pi}_{\delta}^{m}\right) \stackrel{d}{=}\left(\pi_{\delta}^{1}, \ldots, \pi_{\delta}^{m}\right)=\Gamma_{\beta, \delta}\left(\pi_{\delta}^{1}, \ldots, \pi_{\delta}^{m}\right)
$$

and

$$
\Gamma_{\alpha}\left(\widetilde{\pi}_{\delta}^{1}, \ldots, \widetilde{\pi}_{\delta}^{m}\right) \stackrel{d}{=} \Gamma_{\alpha}\left(\pi_{\delta}^{1}, \ldots, \pi_{\delta}^{m}\right) .
$$

With our preparations, to verify condition $\left(I_{1}\right)$, it suffices to show:

(1) Show that $\left(\tilde{\pi}_{\delta}^{1}, \ldots, \tilde{\pi}_{\delta}^{m}\right)$ converges as $\delta \rightarrow 0$ in distribution on $\Pi^{m}$ to $m$ independent Brownian motions $\left(\mathcal{B}^{1}, \ldots, \mathcal{B}^{m}\right)$.

(2) Show that $\Gamma_{\alpha}\left(\pi_{\delta}^{1}, \ldots, \pi_{\delta}^{m}\right)$ and $\Gamma_{\beta, \delta}\left(\pi_{\delta}^{1}, \ldots, \pi_{\delta}^{m}\right)$ are close with high probability as $\delta \rightarrow 0$.

(3) Using Step 1 and (2.32), $\Gamma_{\alpha}\left(\pi_{\delta}^{1}, \ldots, \pi_{\delta}^{m}\right)$ converges in distribution to $m$ coalescing Brownian motions $\left(\mathcal{B}_{\text {coal }}^{1}, \ldots, \mathcal{B}_{\text {coal }}^{m}\right)=\Gamma_{\alpha}\left(\mathcal{B}^{1}, \ldots, \mathcal{B}^{m}\right)$ with the correct starting points. Combining Step 2 and (2.31) then yields the claim. 
Step 1: Let us verify that

$$
\left(\tilde{\pi}_{\delta}^{1}, \ldots, \tilde{\pi}_{\delta}^{m}\right) \underset{\delta \rightarrow 0}{\stackrel{d}{\longrightarrow}}\left(\mathcal{B}^{1}, \ldots, \mathcal{B}^{m}\right)
$$

where $\mathcal{B}^{1}, \ldots, \mathcal{B}^{m}$ are independent Brownian motions and $\mathcal{B}^{i}$ starts from $y_{i}$. Obviously, any limit will have the correct starting points by construction. To identify the limit, we essentially apply the quenched CLT from Birkner et al. (2013) m times, but we have to be a little careful because the rescaled starting points $y_{\delta, i}$ might be inside a "hole" of the cluster $\mathcal{C}$.

Using Birkner et al. (2013, Theorem 1.1, Remark 1.5) we know that for every $(x, n) \in \mathbb{Z} \times \mathbb{Z}$ the diffusively rescaled random walk $\pi_{\delta}^{(x, n)}$ converges weakly under $\mathbb{P}\left(\cdot \mid B_{(x, n)}\right)$ to a Brownian motion, where $B_{(x, n)}$ is the event that $(x, n)$ is connected to infinity. Define $G_{(x, n)}$ to be the event that the quenched functional central limit theorem holds for a path starting in $(x, n)$. Birkner et al. (2013, Theorem 1.1, Theorem 1.4) yields $\mathbb{P}\left(G_{(x, n)} \mid B_{(x, n)}\right)=1$, hence

$$
G:=\bigcap_{(x, n) \in \mathbb{Z}^{2}}\left(G_{(x, n)} \cup\left(B_{(x, n)}\right)^{c}\right)
$$

satisfies $\mathbb{P}(G)=1$ since the complement is a countable union of null sets. Thus up to a $\mathbb{P}$-null set either $(x, n) \in \mathbb{Z} \times \mathbb{Z}$ is not connected to infinity or the quenched functional central limit theorem holds in $(x, n)$. Keeping this in mind, in order to prove the claim of Step 1, is remains to show that

$$
\frac{c\left(y_{\delta, i}\right) \delta}{v} \underset{\delta \downarrow \infty}{\longrightarrow} x_{i} \quad \text { in probability }
$$

where $c((x, n))=\max \{y \leqslant x:(y, n) \in \mathcal{C}\}$ as defined in (1.8).

According to Durrett (1984, Section 10, in particular Eq. (5) on p. 1029) we know that there exist $K, C>0$ such that

$$
\mathbb{P}(|x-c((x, m))| \geqslant K \log (1 / \delta)) \leqslant C \delta^{2} \quad \text { for all }(x, m) \in \mathbb{Z} \times \mathbb{Z} \text { and } \delta \in(0,1) .
$$

The bound (2.35) on the probability of holes of order $\approx \log (1 / \delta)$ to occur implies

$$
\mathbb{P}\left(\left|x_{i}-\frac{c\left(y_{\delta, i}\right) \delta}{v}\right|>\varepsilon\right)=\mathbb{P}\left(\left|x_{i} v \delta^{-1}-c\left(y_{\delta, i}\right)\right|>\frac{\varepsilon v}{\delta}\right) \longrightarrow 0 \quad \text { as } \delta \downarrow 0
$$

for every $\varepsilon>0$ and $i=1, \ldots, m$, from which (2.34) and thus (2.33) follow.

Step 2: Let us write $\left(\pi_{\delta, \alpha}^{1}, \ldots, \pi_{\delta, \alpha}^{m}\right)=\Gamma_{\alpha}\left(\pi_{\delta}^{1}, \ldots, \pi_{\delta}^{m}\right)$ and recall from (2.31) that $\left.\overline{\left(\pi_{\delta}^{1}, \ldots\right.}, \pi_{\delta}^{m}\right)=\Gamma_{\beta, \delta}\left(\pi_{\delta}^{1}, \ldots, \pi_{\delta}^{m}\right)$. We metrize $\Pi^{m}$ with the product metric $d^{* m}$ based on $d(\cdot, \cdot)$ from $(1.6)$.

We claim that for every $\varepsilon>0$,

$$
\mathbb{P}\left(d^{* m}\left(\left(\pi_{\delta, \alpha}^{1}, \ldots, \pi_{\delta, \alpha}^{m}\right),\left(\pi_{\delta}^{1}, \ldots, \pi_{\delta}^{m}\right)\right) \geqslant \varepsilon\right) \underset{\delta \rightarrow 0}{\longrightarrow} 0
$$

(comparing with the definition of $d$ in (1.6), we leave the dependence on the starting times implicit here).

Define a new metric

$$
\bar{d}\left((f, \sigma),\left(g, \sigma^{\prime}\right)\right):=\left|\sigma-\sigma^{\prime}\right| \vee \sup _{t \in \mathbb{R}}\left|f(t \vee \sigma)-g\left(t \vee \sigma^{\prime}\right)\right|
$$


on $\Pi$ and analogously $\bar{d}^{* m}$ on $\Pi^{m}$. We have $d\left(\left(f_{1}, t_{1}\right),\left(f_{2}, t_{2}\right)\right) \leqslant \bar{d}\left(\left(f_{1}, t_{1}\right),\left(f_{2}, t_{2}\right)\right)$ for all $\left(f_{1}, t_{1}\right),\left(f_{2}, t_{2}\right) \in \Pi$, since $\tanh (\cdot)$ is Lipschitz continuous with Lipschitz constant one. Therefore in order to prove (2.36) its enough to show that

$$
\begin{aligned}
\mathbb{P}\left(\overline { d } ^ { * m } \left[\left(\left(\pi_{\delta, \alpha}^{1},\left\lfloor\delta^{-2} t_{1}\right\rfloor\right), \ldots,\left(\pi_{\delta, \alpha}^{m},\left\lfloor\delta^{-2} t_{m}\right\rfloor\right)\right),\right.\right. \\
\left.\left.\left(\left(\pi_{\delta}^{1},\left\lfloor\delta^{-2} t_{1}\right\rfloor\right), \ldots,\left(\pi_{\delta}^{m},\left\lfloor\delta^{-2} t_{m}\right\rfloor\right)\right)\right] \geqslant \varepsilon\right) \underset{\delta \rightarrow 0}{\longrightarrow} 0 .
\end{aligned}
$$

We prove (2.37) by induction over $m$.

Let $m=2$. Since $\pi_{\delta, \alpha}^{1}=\pi_{\delta}^{1}$ by construction we get that

$$
\begin{aligned}
& \bar{d}^{* 2}\left[\left(\left(\pi_{\delta, \alpha}^{1},\left\lfloor\delta^{-2} t_{1}\right\rfloor\right),\left(\pi_{\delta, \alpha}^{2},\left\lfloor\delta^{-2} t_{2}\right\rfloor\right)\right),\left(\left(\pi_{\delta}^{1},\left\lfloor\delta^{-2} t_{1}\right\rfloor\right),\left(\pi_{\delta}^{2},\left\lfloor\delta^{-2} t_{2}\right\rfloor\right)\right)\right] \\
& \left.\quad=\bar{d}\left[\left(\pi_{\delta, \alpha}^{2},\left\lfloor\delta^{-2} t_{2}\right\rfloor\right),\left(\pi_{\delta}^{2}, \mid \delta^{-2} t_{2}\right\rfloor\right)\right] \\
& \quad \leqslant \delta \sup \left\{\left|\pi^{y_{\delta, 1}}(t)-\pi^{y_{\delta, 2}}(t)\right|: T_{\text {near }}^{y_{\delta, 1}, y_{\delta, 2}} \leqslant t \leqslant T_{\text {meet }}^{y_{\delta, 1}, y_{\delta, 2}}\right\}
\end{aligned}
$$

(recall $T_{\text {near }}^{y_{\delta, 1}, y \delta, 2}$ from (2.23) and $T_{\text {meet }}^{y \delta, 1, y \delta, 2}$ from (2.24)). The bound in (2.38) holds because $\pi^{z}$ 's are linear interpolations of discrete walks with steps from $\{-1,0,1\}$ and by definition of the merging rule $\Gamma_{\alpha}, \pi_{\delta, \alpha}^{2}(t)=\pi_{\delta}^{2}(t)$ for $t<T_{\text {near }}^{y_{\delta, 1}, y \delta, 2}$. (2.38) and (2.26) from Lemma 2.6 imply (2.37) for $m=2$.

Now let $m>2$. Here, we can argue essentially analogously to Newman et al. (2005, p. 45). There are two possibilities for the event in (2.37) to occur.

The first possibility is that a "wrong" $(\alpha$-)coalescing event occurs, which means that for some $k$ and $i<j$ a path $\pi_{1}^{l}, l<i$ coalesces or changes its relative order with $\pi_{1}^{i}$ after time $\tau_{\alpha}^{k}=T_{\alpha}^{i, j}$ and before time $T_{\beta}^{i, j}$ (where there is then no need for $\pi_{1}^{l}$ and $\pi_{1}^{j}$ to coalesce "soon" since their paths did not cross before). Let us consider this case.

Using Step 1 (see also Birkner et al. (2013, Theorem 1.3, Remark 1.5, Remark 3.11) ) and (2.32) together with the fact that $\mathcal{L}\left(\mathcal{B}^{1}, \ldots, \mathcal{B}^{m}\right)$ has full measure on the set of continuity points of the mapping $\Gamma_{\alpha}, \Gamma_{\alpha}\left(\widetilde{\pi}_{\delta}^{1}, \ldots, \widetilde{\pi}_{\delta}^{m}\right)$ converges in distribution on $\Pi^{m}$ to $m$ coalescing Brownian motions

$$
\left(\mathcal{B}_{\text {coal }}^{1}, \ldots, \mathcal{B}_{\text {coal }}^{m}\right)=\Gamma_{\alpha}\left(\mathcal{B}^{1}, \ldots, \mathcal{B}^{m}\right)
$$

with the correct starting points. Write $\left\{T_{\alpha, \delta}^{i, j}\right\}_{1 \leqslant i, j \leqslant m}$ for the coalescence times of $\left(\tilde{\pi}_{\delta, \alpha}^{1}, \ldots, \tilde{\pi}_{\delta, \alpha}^{m}\right)=\Gamma_{\alpha}\left(\tilde{\pi}_{\delta}^{1}, \ldots, \tilde{\pi}_{\delta}^{m}\right)$ and $\left\{T_{\beta, \delta}^{i, j}\right\}_{1 \leqslant i, j \leqslant m}$ for the coalescence times of $\left(\tilde{\pi}_{\delta, \beta}^{1}, \ldots, \tilde{\pi}_{\delta, \beta}^{m}\right)=\Gamma_{\beta}\left(\tilde{\pi}_{\delta}^{1}, \ldots, \tilde{\pi}_{\delta}^{m}\right)$. We thus obtain for all $i \neq j \leqslant m$

$$
\left(T_{\alpha, \delta}^{i, j}\right)_{1 \leqslant i \neq j \leqslant m} \underset{\delta \rightarrow 0}{\stackrel{d}{\longrightarrow}}\left(\tau^{i, j}\right)_{1 \leqslant i \neq j \leqslant m}
$$

where $\tau^{i, j}$ is the coalescence time (and indeed also the first crossing time) of $\mathcal{B}_{\text {coal }}^{i}$ and $\mathcal{B}_{\text {coal }}^{j}$. Note that almost surely, $\left(\mathcal{B}_{\text {coal }}^{1}, \ldots, \mathcal{B}_{\text {coal }}^{m}\right)=\Gamma_{\alpha}\left(\mathcal{B}^{1}, \ldots, \mathcal{B}^{m}\right)$ arises via $m-1$ distinct coalescence events at a.s. distinct times.

Furthermore, Lemma 2.6 shows that for every $\varepsilon>0$, the events

$$
A_{\delta}(\varepsilon):=\bigcap_{1 \leqslant i<j \leqslant m}\left\{T_{\text {meet }}^{y_{\delta, i}, y_{\delta, j}}-T_{\text {near }}^{y_{\delta, i}, y_{\delta, j}}<\frac{\varepsilon}{\delta^{2}}\right\}
$$

satisfy $\lim _{\delta \downarrow 0} \mathbb{P}\left(A_{\delta}(\varepsilon)\right)=1$. On the event

$$
A_{\delta}(\varepsilon) \cap\left\{\inf \left(\left\{\left|T_{\alpha, \delta}^{i, j}-T_{\alpha, \delta}^{i^{\prime}, j^{\prime}}\right|: 1 \leqslant i, j, i^{\prime}, j^{\prime} \leqslant m,(i, j) \neq\left(i^{\prime}, j^{\prime}\right)\right\} \backslash\{0\}\right)>2 \varepsilon\right\}
$$


we have

$$
\max _{1 \leqslant i<j \leqslant m}\left|T_{\alpha, \delta}^{i, j}-T_{\beta, \delta}^{i, j}\right| \leqslant \varepsilon .
$$

Since $\varepsilon>0$ is arbitrary, we have in fact

$$
\max _{1 \leqslant i<j \leqslant m}\left|T_{\alpha, \delta}^{i, j}-T_{\beta, \delta}^{i, j}\right| \underset{\delta \rightarrow 0}{\longrightarrow} 0 \text { in probability. }
$$

But then the probability of a "wrong" coalescing event tends to zero, since all the crossing times of the Brownian motions are a.s. distinct.

The second possibility for the event in (2.37) to occur is that there is "too much" time between the crossing and the coalescence. "Too much" time means there is a positive probability that at least one pair of the random walks needs more than $\varepsilon / \delta^{2}$ steps to coalesce after their paths crossed, for some $\varepsilon>0$, which would allow $\max _{1 \leqslant i \neq j \neq m} \sup _{t}\left|\pi_{\delta, \alpha}^{i}(t)-\pi_{\delta}^{i}(t)\right|$ to remain "macroscopic". This is ruled out by an argument similar to the one above, note that again by Lemma 2.6, the events

$$
A_{\delta}^{\prime}(\varepsilon):=\bigcap_{1 \leqslant i<j \leqslant m}\left\{\sup \left\{\left|\pi^{\delta, i}(t)-\pi^{y_{\delta, j}}(t)\right|: T_{\text {near }}^{y_{\delta, i}, y_{\delta, j}} \leqslant t \leqslant T_{\text {meet }}^{y_{\delta, i}, y_{\delta, j}}\right\}<\frac{\varepsilon}{\delta}\right\}
$$

satisfy $\lim _{\delta \downarrow 0} \mathbb{P}\left(A_{\delta}^{\prime}(\varepsilon)\right)=1$ for every $\varepsilon>0$. Thus, the proof of (2.37) for $m>2$ is completed.

Step 3 (Verification of $\left.\left(I_{1}\right)\right)$ : Combine $(2.33),(2.32)$ and $(2.36)$ to see that

$$
\left(\pi_{\delta}^{1}, \ldots, \pi_{\delta}^{m}\right) \underset{\delta \rightarrow 0}{\stackrel{d}{\longrightarrow}}\left(\mathcal{B}_{\text {coal }}^{1}, \ldots, \mathcal{B}_{\text {coal }}^{m}\right)
$$

Remark 2.8. An alternative route to (2.40) would be to use the characterization of the law of coalescing Brownian motions (viewed as the special case of $\theta$-sticky Brownian motions with $\theta=0$ ) as the unique solution of a martingale problem from Howitt (2007, Theorem 76). See also Howitt and Warren (2009, Theorem 2.1) and the discussion in Schertzer et al. (2017, Section 5), Schertzer et al. (2014, Appendix A) as well as Schertzer and Sun (2018, Appendix A). In fact, this would require to check that for any weak limit point $\left(\tilde{\mathcal{B}}^{1}, \ldots, \tilde{\mathcal{B}}^{m}\right)$ of $\left(\pi_{\delta_{n}}^{1}, \ldots, \pi_{\delta_{n}}^{m}\right)$ with $\delta_{n} \rightarrow 0$, the following holds: Let $\mathcal{F}=\left(\mathcal{F}_{t}\right)_{t \in \mathbb{R}}$ with $\mathcal{F}_{t}=\sigma\left(\left(\tilde{\mathcal{B}}^{i}(s \wedge t)\right)_{s \geqslant t_{i}}\right.$, for $i$ s.th. $\left.t_{i} \leqslant t\right)$ be the joint filtration generated by $\tilde{\mathcal{B}}^{1}, \ldots, \tilde{\mathcal{B}}^{m}$. Then 1 . each $\tilde{\mathcal{B}}^{i}$ is an $\mathcal{F}$-Brownian motion starting from space-time point $y_{i}=\left(x_{i}, t_{i}\right)$, and 2 . each pair $\left(\tilde{\mathcal{B}}^{i}, \tilde{\mathcal{B}}^{j}\right), i \neq j$ is distributed as a pair of coalescing Brownian motions (w.r.t. the filtration $\mathcal{F}$ ).

The fact that each $\tilde{\mathcal{B}}^{i}$ individually is a Brownian motion follows immediately from the central limit theorem proved in Birkner et al. (2013) together with Step 1 above and the fact that $\left(\tilde{\mathcal{B}}^{i}, \tilde{\mathcal{B}}^{j}\right)$ are coalescing Brownian motions was checked in Step 2, case $m=2$ above. However, in our set-up it appears quite cumbersome to verify directly that these properties also hold with respect to the larger joint filtration $\mathcal{F}$. The natural way to such a result is to consider $\left(\pi^{y_{\delta, 1}}, \ldots, \pi^{y_{\delta, m}}\right)$ along joint regeneration times (cf Remark 2.3). This yields a Markov chain on $\mathbb{Z}^{m}$, then one would need a suitable $m$-coordinate analogue of Lemma 2.2 and therewith implement a martingale plus remainder term decomposition of the coordinates of this chain analogous to the construction in Birkner et al. (2013, Section 3.4) to conclude. In our view, spelling out the details would be more laborious than the approach discussed above. On the other hand, using (2.40) we can conclude that properties 1 . and 2. discussed above do hold. 


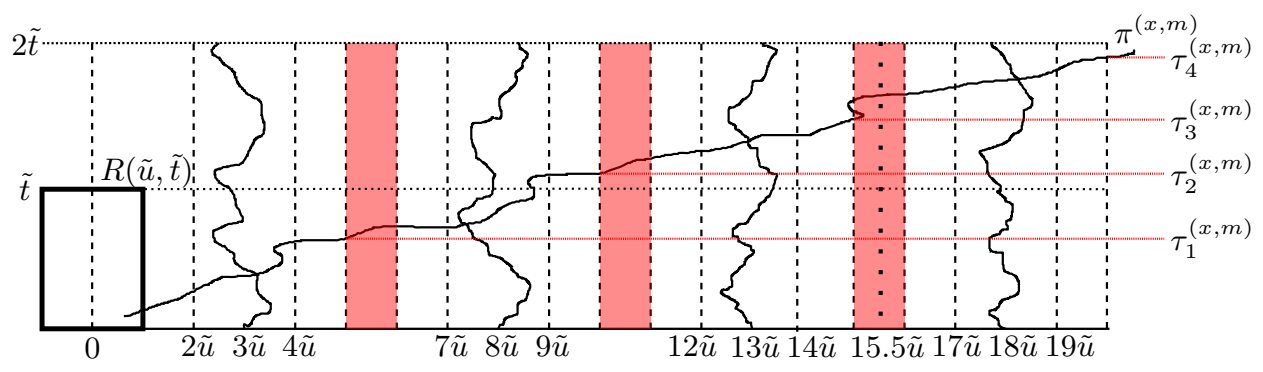

Figure 2.1. An illustration of (part of) the event $\boldsymbol{\Gamma} \in A_{\tilde{t}, \tilde{u}}^{+}(0,0) \cap \bigcap_{i=1}^{4} B_{i}$

2.3.3. Checking condition $\left(T_{1}\right)$. Let $A_{t, u}^{+}\left(x_{0}, t_{0}\right)$ be the set of $K \in \mathcal{H}$ which contain a path touching both $R\left(x_{0}, t_{0}, u, t\right)$ and the right boundary of the bigger rectangle $R\left(x_{0}, t_{0}, 20 u, 2 t\right)$. Similarly we define $A_{t, u}^{-}\left(x_{0}, t_{0}\right)$ as the event that the path hits the left boundary of the bigger rectangle. If a variable is diffusively scaled we will add a " $\sim$ " to it, where $\tilde{t}=t \delta^{-2}$ if $t$ is a time variable and $\tilde{x}=x /(v \delta)$ if $x$ is a space-variable. In order to verify condition $\left(T_{1}\right)$ it is enough to show that for every $u \in(0, \infty)$

$$
t^{-1} \limsup _{\delta \rightarrow 0} \mu_{1}\left(A_{\tilde{t}, \tilde{u}}^{+}(0,0)\right) \longrightarrow 0 \text { as } t \rightarrow 0^{+},
$$

where we omitted the sup over $\left(x_{0}, t_{0}\right)$ from condition $\left(T_{1}\right)$ because of the spatial invariance of $\mu_{1}=\mathbb{P} \circ\left(S_{v, 1} \boldsymbol{\Gamma}\right)^{-1}$. (2.41) implies $\left(T_{1}\right)$ since $A_{t, u}\left(x_{0}, t_{0}\right)=$ $A_{t, u}^{+}\left(x_{0}, t_{0}\right) \cup A_{t, u}^{-}\left(x_{0}, t_{0}\right)$ and $\mu_{1}\left(A_{\tilde{t}, \tilde{u}}^{-}(0,0)\right)$ can be estimated completely analogously (in fact, we even have $\mu_{1}\left(A_{\tilde{t}, \tilde{u}}^{-}(0,0)\right)=\mu_{1}\left(A_{\tilde{t}, \tilde{u}}^{+}(0,0)\right)$ by symmetry).

We will show that for every fixed $u>0, \limsup _{\delta \rightarrow 0} \mu_{1}\left(A_{\tilde{t}, \tilde{u}}^{+}(0,0)\right)$ is in $\mathbf{o}(t)$. Let $u>0$ and define $x_{1, \delta}:=\lfloor 3 \tilde{u}\rfloor, x_{2, \delta}:=\lfloor 8 \tilde{u}\rfloor, x_{3, \delta}:=\lfloor 13 \tilde{u}\rfloor$ and $x_{4, \delta}:=\lfloor 18 \tilde{u}\rfloor$ with $\tilde{u}=v u \delta^{-1}$. We are interested in the paths $\pi^{x_{i, \delta}}:=\pi^{\left(x_{i, \delta}, 0\right)}, i=1,2,3,4$.

We denote by $B_{i}$ the event that $\pi^{x_{i, \delta}}$ stays within distance $\tilde{u}$ of $x_{i, \delta}$ up to time $2 \tilde{t}$. For a fixed $(x, m) \in R(\tilde{u}, \tilde{t}):=R(0,0, \tilde{u}, \tilde{t})$ denote the times when the random walker $\pi^{(x, m)}$ first exceeds $5 \tilde{u}, 10 \tilde{u}, 15 \tilde{u}$ and $20 \tilde{u}$ by $\tau_{1}^{(x, m)}, \tau_{2}^{(x, m)}, \tau_{3}^{(x, m)}$ and $\tau_{4}^{(x, m)}$. Furthermore define $\tau_{0}^{(x, m)}=0$ and $\tau_{5}^{(x, m)}=2 \tilde{t}$. Denote by $C_{i}(x, m)$ the event that $\pi^{(x, m)}$ does not coalesce with $\pi^{x_{i, \delta}}$ before time $2 \tilde{t}$. We assume that $\tilde{t} \in \mathbb{Z}$, if not we replace $\tilde{t}$ by $\lceil\tilde{t}\rceil$. We estimate the probability in (2.41) in the following way (see Figure 2.1):

$$
\begin{aligned}
\mu_{1}\left(A_{\tilde{t}, \tilde{u}}^{+}(0,0)\right) \leqslant & \mu_{1}\left(\bigcup_{i=1}^{4} B_{i}^{c}\right) \\
& +\mu_{1}\left(\bigcap_{i=1}^{4} B_{i} \cap \bigcup_{(x, m) \in R(\tilde{u}, \tilde{t})}\left(\bigcap_{i=1}^{4} C_{i}(x, m) \cap\left\{\tau_{4}^{(x, m)}<2 \tilde{t}\right\}\right)\right)
\end{aligned}
$$

We estimate the terms $(*)$ and $(* *)$ separately. We have

$$
\limsup _{\delta \rightarrow 0} \mu_{1}\left(\bigcup_{i=1}^{4} B_{i}^{c}\right) \leqslant 4 \limsup _{\delta \rightarrow 0} \mu_{1}\left(B_{1}^{c}\right)
$$




$$
=4 \mathbb{P}\left(\sup _{s \in[0, t]}\left|B_{s}\right|>u\right) \leqslant 16 e^{-\frac{u^{2}}{2 t}} \in \mathbf{o}(t) \text { as } t \downarrow 0
$$

where $B$ is a standard Brownian motion.

The second term $(* *)$ can be estimated as follows

$$
(* *) \leqslant \sum_{\substack{x \in[-\tilde{u}, \tilde{u}] \cap \mathbb{Z} \\ m \in[0, \tilde{t}] \cap \mathbb{Z}}} \mu_{1}\left(\bigcap_{i=1}^{4} B_{i} \cap \bigcap_{i=1}^{4} C_{i}(x, m) \cap\left\{\tau_{4}^{(x, m)}<2 \tilde{t}\right\}\right)
$$

Now we change our point of view on the problem. From now on we come back to the discrete structure and are only interested in the values of the random walk path at simultaneous regeneration times $T_{j}^{\text {sim }}$ (of the five random walks), recall the discussion in Section 2.1 and especially Remark 2.3.

Denote by $\theta_{i}$ the first simultaneous regeneration time when $\pi^{(x, m)}(n)-\pi^{x_{i, \delta}}(n)>$ 0 . Furthermore let $\widehat{B}_{i}$ the event that $\pi^{x_{i, \delta}}$ stays within distance $\tilde{u}$ of $x_{i, \delta}$ at simultaneous regeneration times up to time $2 \tilde{t}$ and denote by $\widehat{C}_{i}(x, m)$ the event that $\pi^{(x, m)}$ does not coincide with $\pi^{x_{i, \delta}}$ at simultaneous regeneration times before time $2 \tilde{t}$. In analogy to the previous notation let $\hat{\tau}_{i}^{(x, m)}$ be the first time that a simultaneous regeneration event occurs after the the random walk path $\pi^{(x, m)}$ exceeds $(5 \cdot i) \tilde{u}$. Only considering the random walks at simultaneous regeneration times, we can for every $\varepsilon>0$ estimate a single summand of the sum above by

$$
\begin{aligned}
& \mu_{1}\left(\bigcap_{i=1}^{4} B_{i} \cap \bigcap_{i=1}^{4} C_{i}(x, m) \cap\left\{\tau_{4}^{(x, m)}<2 \tilde{t}\right\}\right) \\
& \leqslant \mu_{1}\left(\bigcap_{i=1}^{4} \widehat{B}_{i} \cap \bigcap_{i=1}^{4} \widehat{C}_{i}(x, m) \cap\left\{\hat{\tau}_{4}^{(x, m)}<(2+\varepsilon) \tilde{t}\right\}\right) \\
& \quad+\mathbb{P}(\text { no simultaneous regeneration between time } 2 \tilde{t} \text { and time }(2+\varepsilon) \tilde{t}) \\
& \leqslant \mu_{1}\left(\bigcap_{i=1}^{4} \hat{B}_{i} \cap \bigcap_{i=1}^{4} \widehat{C}_{i}(x, m) \cap\left\{\hat{\tau}_{4}^{(x, m)}<(2+\varepsilon) \tilde{t}\right\} \cap\left\{T_{\theta_{4}}^{\operatorname{sim}}-T_{\theta_{4}-1}^{\operatorname{sim}}<C \log \left(\frac{1}{\delta}\right)\right\}\right) \\
& \quad+\delta^{4}+2 \tilde{t} C e^{-c \varepsilon \tilde{t}}
\end{aligned}
$$

by using exponential tail bounds for increments of $T_{\ell}^{\mathrm{sim}}-T_{\ell-1}^{\mathrm{sim}}$, see Remark 2.3. Here we use that $\theta_{4}$ is a stopping time for the joint regeneration construction of the five walks and that we can choose $C$ so large that

$$
\mathbb{P}\left(T_{\theta_{4}}^{\mathrm{sim}}-T_{\theta_{4}-1}^{\mathrm{sim}} \geqslant C \log \left(\frac{1}{\delta}\right)\right) \leqslant \delta^{4} .
$$

Furthermore, the probability that no simultaneous regeneration occurs between time $2 \tilde{t}$ and time $(2+\varepsilon) \tilde{t}$ is bounded from above by

$$
\sum_{\ell=1}^{\lceil 2 \tilde{t}\rceil} \mathbb{P}\left(T_{\ell}^{\operatorname{sim}}-T_{\ell-1}^{\operatorname{sim}}>\varepsilon \tilde{t}\right) \leqslant 2 \tilde{t} C e^{-c \varepsilon \tilde{t}}=2 t \delta^{-2} e^{-c \varepsilon t / \delta^{2}}=O\left(t \delta^{4}\right) .
$$

Now by the regeneration structure, the only information we gained about the "future" after time $T_{\theta_{4}}^{s i m}$ of the cluster is that each of the five random walks is at a space-time-point that is connected to infinity. Therefore, without changing the joint distribution, the future of the cluster can be replaced by some identical copy in which all the points the random walks sit in are connected to infinity. By a coupling 
argument as in the proof of Lemma 3.4 in Birkner et al. (2013), the cluster to the right of the middle line of the third red bar (at horizontal coordinate $x=15.5 \tilde{u}$, see Figure 2.1) can be replaced by an independent copy and the resulting law on configurations strictly to the right of this third red bar (i.e., $x>16 \tilde{u}$ ) has total variation distance at most $2 \tilde{t} C e^{-c \tilde{u}}$ to the original law. Thus

$$
\begin{gathered}
\mu_{1}\left(\bigcap_{i=1}^{4} \widehat{B}_{i} \cap \bigcap_{i=1}^{4} \widehat{C}_{i}(x, m) \cap\left\{\hat{\tau}_{4}^{(x, m)}<(2+\varepsilon) \tilde{t}\right\} \cap\left\{T_{\theta_{4}}^{\operatorname{sim}}-T_{\theta_{4}-1}^{\operatorname{sim}}<C \log \left(\frac{1}{\delta}\right)\right\}\right) \\
\leqslant \mu_{1}\left(\bigcap_{i=1}^{3} \widehat{B}_{i} \cap \bigcap_{i=1}^{3} \widehat{C}_{i}(x, m) \cap\left\{\hat{\tau}_{3}^{(x, m)}<(2+\varepsilon) \tilde{t}\right\}\right) \\
\quad \times \sup _{|y| \leqslant C \log (1 / \delta)} \mathbb{P}_{y}^{\text {joint }}(\hat{D} \text { hits } \tilde{u} \text { before } 0)+2 \tilde{t} C e^{-c \tilde{u}} .
\end{gathered}
$$

We use here that the difference between $\pi^{(x, m)}$ and $\pi^{x_{4, \delta}}$, running on an independent copy of the percolation cluster (and observed along its regeneration times), behaves like the Markov chain $\widehat{D}$ from Section 2.1 and the proof of Lemma 2.4. Remark 2.5 gives in particular

$$
\sup _{|y| \leqslant C \log (1 / \delta)} \mathbb{P}_{y}^{\text {joint }}(\hat{D} \text { hits } \tilde{u} \text { before } 0) \leqslant c \frac{C \log (1 / \delta)}{\tilde{u}}=C^{\prime} \delta \log (1 / \delta)
$$

with $C^{\prime}=C^{\prime}(u)<\infty$.

Combining the above and iterating we get

$$
\mu_{1}\left(\bigcap_{i=1}^{4} B_{i} \cap \bigcap_{i=1}^{4} C_{i}(x, m) \cap\left\{\tau_{4}^{(x, m)}<2 \tilde{t}\right\}\right) \leqslant\left(2 C^{\prime} \delta \log (1 / \delta)\right)^{4} .
$$

Using this, the term $(* *)$ is bounded above by

$$
\begin{aligned}
& \mu_{1}\left(\bigcap_{i=1}^{4} B_{i}, \exists(x, m) \in R(\tilde{u}, \tilde{t}) \text { s.t. } \bigcap_{i=1}^{4} C_{i}(x, m) \text { and } \tau_{4}^{(x, m)}<2 \tilde{t}\right) \\
& \leqslant \sum_{x \in[-\tilde{u}, \tilde{u}] \cap \mathbb{Z}} \sum_{m \in[0, \tilde{t}] \cap \mathbb{Z}}\left(2 C^{\prime} \delta \log (1 / \delta)\right)^{4} \leqslant\left(2 C^{\prime} \delta \log (1 / \delta)\right)^{4} \cdot 2 \tilde{u} \tilde{t} \leqslant C(u) t \delta(\log (1 / \delta))^{4}
\end{aligned}
$$

This implies that condition (2.41) is satisfied.

2.3.4. Checking condition $\left(B_{1}^{\prime}\right)$. We fix $t>\beta>0$ and $t_{0}, a \in \mathbb{R}$. We want to show that for each $\varepsilon^{\prime}>0$ there exists $\varepsilon>0$ independent of $t, t_{0}$ and $a$, such that

$$
\mu_{\delta}\left(\eta\left(t_{0}, t ; a-\varepsilon, a+\varepsilon\right)>1\right)=\mu_{1}(\eta(\tilde{t} 0, \tilde{t} ; \tilde{a}-\tilde{\varepsilon}, \tilde{a}+\tilde{\varepsilon})>1)<\varepsilon^{\prime},
$$

for all $\delta>0$ sufficiently small. First we assume that $\tilde{t}_{0}=n_{0} \in \mathbb{Z}$. In this case only paths that start from the interval $[\tilde{a}-\tilde{\varepsilon}, \tilde{a}+\tilde{\varepsilon}] \cap \mathbb{Z}$ at time $n_{0}$ are counted by $\eta$. Therefore

$$
\begin{aligned}
& \mu_{1}\left(\eta\left(n_{0}, \tilde{t} ; \tilde{a}-\tilde{\varepsilon}, \tilde{a}+\tilde{\varepsilon}\right)>1\right) \\
& \leqslant \sum_{\{x, x+1\} \subset[\tilde{a}-\tilde{\varepsilon}, \tilde{a}+\tilde{\varepsilon}] \cap \mathbb{Z}} \mathbb{P}\left(\pi^{\left(x, n_{0}\right)}(k) \neq \pi^{\left(x+1, n_{0}\right)}(k) \text { for all } k \in\left[n_{0}, n_{0}+\lfloor\tilde{t}]\right]\right) .
\end{aligned}
$$

By Lemma 2.4 we get that

$$
\mathbb{P}\left(\pi^{\left(x, n_{0}\right)}(k) \neq \pi^{\left(x+1, n_{0}\right)}(k) \text { for all } k \in\left[n_{0}, n_{0}+[\tilde{t}]\right]\right) \leqslant \frac{C}{\sqrt{\tilde{t}}}
$$


for some large constant $C$ and

$$
\mu_{1}\left(\eta\left(n_{0}, \tilde{t} ; \tilde{a}-\tilde{\varepsilon}, \tilde{a}+\tilde{\varepsilon}\right)>1\right) \leqslant \frac{2 \tilde{\varepsilon} C}{\sqrt{\tilde{t}}} \leqslant \frac{2 v \varepsilon C}{\sqrt{t}} \leqslant \frac{2 v \varepsilon C}{\sqrt{\beta}},
$$

which is smaller than $\varepsilon^{\prime}$ if $\varepsilon<\frac{\varepsilon^{\prime} \sqrt{\beta}}{2 v C}$.

If $\tilde{t}_{0} \in\left(n_{0}, n_{0}+1\right)$ for some $n_{0} \in \mathbb{N}$, it is enough to show that $\mu_{1}\left(\eta\left(\tilde{t} \tilde{t}_{0}, \tilde{t} ; \tilde{a}-2 \tilde{\varepsilon}, \tilde{a}+\right.\right.$ $2 \tilde{\varepsilon})>1)<\varepsilon^{\prime}$, which is true by similar estimates as above.

2.3.5. Checking condition $\left(E_{1}^{\prime}\right)$. In order to verify condition $\left(E_{1}^{\prime}\right)$ we need to prove a statement similar to Lemma 6.2 in Newman et al. (2005) which is formulated in Lemma 2.10 below. This can be done by adapting Lemma 2.7 in Newman et al. (2005) to our case (see Lemma 2.9 below). The rest of the proof follows by more general results, proved in Newman et al. (2005, Section 6)) and does not need adaptation.

Lemma 2.9. Recall the collection of paths $\boldsymbol{\Gamma}$ from (1.9). For $A \subset \mathbb{Z}$ and $m, n \in$ $\mathbb{N}, m>n$, we define

$$
\boldsymbol{\Gamma}_{m}^{A, n}:=\left\{\pi^{(x, n)}(m): x \in A,(x, n) \in \mathcal{C}\right\} .
$$

If $n=0$ we simply write $\boldsymbol{\Gamma}_{m}^{A}:=\boldsymbol{\Gamma}_{m}^{A, 0}$. Then

$$
p_{m}:=\mathbb{P}\left(0 \in \Gamma_{m}^{\mathbb{Z}}\right) \leqslant \frac{C}{\sqrt{m}},
$$

for some constant $C$ independent of time.

Proof: Pick $M \in \mathbb{N}$. Let $B_{M}:=\{0,1, \ldots, M-1\}$ and in order to simplify notation define

$$
\boldsymbol{\Gamma}_{m}^{A}(x):=\left\{\begin{array}{lc}
1, & \text { if } x \in \boldsymbol{\Gamma}_{m}^{A} \\
0, & \text { otherwise }
\end{array}\right.
$$

for $A \subset \mathbb{Z}$. Using the translation invariance of $\mathbb{P}$ we obtain

$$
e_{m}\left(B_{M}\right):=\mathbb{E}\left[\left|\boldsymbol{\Gamma}_{m}^{\mathbb{Z}} \cap B_{M}\right|\right]=\mathbb{E}\left[\sum_{x \in B_{M}} \boldsymbol{\Gamma}_{m}^{\mathbb{Z}}(x)\right]=\sum_{x \in B_{M}} \mathbb{E}\left[\boldsymbol{\Gamma}_{m}^{\mathbb{Z}}(x)\right]=p_{m} \cdot M .
$$

Furthermore,

$$
e_{m}\left(B_{M}\right) \leqslant \sum_{k \in \mathbb{Z}} \mathbb{E}\left[\left|\boldsymbol{\Gamma}_{m}^{B_{M}+k M} \cap B_{M}\right|\right]=\sum_{k \in \mathbb{Z}} \mathbb{E}\left[\left|\boldsymbol{\Gamma}_{m}^{B_{M}} \cap\left(B_{M}-k M\right)\right|\right]=\mathbb{E}\left[\left|\boldsymbol{\Gamma}_{m}^{B_{M}}\right|\right] .
$$

Now the difference $M-\left|\boldsymbol{\Gamma}_{m}^{B_{M}}\right|$ is larger than the number of nearest neighbour pairs that coalesced before time $m$. Using the translation invariance of $\mathbb{P}$ again we get that

$$
\begin{aligned}
\mathbb{E}\left[M-\left|\boldsymbol{\Gamma}_{m}^{B_{M}}\right|\right] & \geqslant \sum_{x=0}^{M-2} \mathbb{E}\left[\mathbb{1}_{\left\{\pi^{(x, 0)}(t)=\pi^{(x+1,0)}(t) \text { for some } t \in\{1,2, \ldots, m\}\right\}}\right] \\
& =(M-1) \mathbb{P}\left[\pi^{(0,0)}(t)=\pi^{(1,0)}(t) \text { for some } t \in\{1,2, \ldots, m\}\right]
\end{aligned}
$$

Lemma 2.4 gives

$$
\begin{aligned}
\mathbb{E}\left[\left|\boldsymbol{\Gamma}_{m}^{B_{M}}\right|\right] & \leqslant M-(M-1) \mathbb{P}\left[\pi^{(0,0)}(t)=\pi^{(1,0)}(t) \text { for some } t \in\{1,2, \ldots, m\}\right] \\
& \leqslant M-(M-1)\left(1-\frac{C}{\sqrt{m}}\right)<1+M \frac{C}{\sqrt{m}}
\end{aligned}
$$


and therefore

$$
p_{m}<\frac{1}{M}+\frac{C}{\sqrt{m}} .
$$

This yields the claim since $M$ can be chosen arbitrarily large.

Now we are ready to prove our analogon of Newman et al. (2005, Lemma 6.2). Recall the notation $\mathcal{X}^{t_{0}^{-}}$from (2.28).

Lemma 2.10. Let $\mathcal{Z}_{t_{0}}$ be a subsequential limit of $\mathcal{X}_{\delta}^{t_{0}^{-}}$, where $\mathcal{X}_{\delta}:=S_{v, \delta} \boldsymbol{\Gamma}$ and let $\varepsilon>0$. The intersection of the paths in $\mathcal{Z}_{t_{0}}$ with the line $\mathbb{R} \times\left\{t_{0}+\varepsilon\right\}$ is almost surely locally finite.

Proof: Let $\mathcal{Z}_{t_{0}}$ be the weak limit of a sequence $\left(\mathcal{X}_{\delta_{n}}^{t_{0}^{-}}\right)_{n}$ and let $\mathcal{Z}_{t_{0}}\left(t_{0}+\varepsilon\right)$ be the intersection of all paths in $\mathcal{Z}_{t_{0}}$ with the line $t_{0}+\varepsilon$; define $\mathcal{X}_{\delta_{n}}^{t_{0}^{-}}\left(t_{0}+\varepsilon\right)$ analogously. Then $\mathcal{Z}_{t_{0}}$ and $\mathcal{X}_{\delta_{n}}^{t_{0}^{-}}\left(t_{0}+\varepsilon\right), n \in \mathbb{N}$ are random variables with values in $\left(\mathcal{P}, \rho_{\mathcal{P}}\right)$, where $\mathcal{P}$ is the space of all compact subsets of $\left(R_{c}^{2}, \rho\right)$, metrized with the induced Hausdorff metric $\rho_{\mathcal{P}}$.

Since for all $a, b \in \mathbb{R}, a<b$ the set $\left\{K \in\left(\mathcal{P}, \rho_{\mathcal{P}}\right):|K \cap(a, b) \times \mathbb{R}| \geqslant k\right\}$ is an open set in $\left(\mathcal{P}, \rho_{\mathcal{P}}\right)$, we get that

$$
\begin{aligned}
\mathbb{E}\left[\left|\mathcal{Z}_{t_{0}}\left(t_{0}+\varepsilon\right) \cap(a, b) \times \mathbb{R}\right|\right] & =\sum_{k=1}^{\infty} \mathbb{P}\left[\left|\mathcal{Z}_{t_{0}}\left(t_{0}+\varepsilon\right) \cap(a, b) \times \mathbb{R}\right| \geqslant k\right] \\
& \leqslant \sum_{k=1}^{\infty} \liminf _{n \longrightarrow \infty} \mathbb{P}\left[\left|\mathcal{X}_{\delta_{n}}^{t_{0}^{-}}\left(t_{0}+\varepsilon\right) \cap(a, b) \times \mathbb{R}\right| \geqslant k\right] \\
& \leqslant \liminf _{n \longrightarrow \infty} \mathbb{E}\left[\left|\mathcal{X}_{\delta_{n}}^{t_{0}^{-}}\left(t_{0}+\varepsilon\right) \cap(a, b) \times \mathbb{R}\right|\right] \leqslant \frac{C(b-a)}{\sqrt{\varepsilon}},
\end{aligned}
$$

where we used the Portmanteau theorem in the second line. The last inequality holds true by Lemma 2.9, since (recall the scaling notation $\tilde{x}$, etc. introduced before $(2.41))$

$$
\begin{aligned}
& \mathbb{E}\left[\left|\mathcal{X}_{\delta}^{t_{0}^{-}}\left(t_{0}+\varepsilon\right) \cap(a, b) \times \mathbb{R}\right|\right] \\
& \leqslant \mathbb{E}\left[\sum_{x \in(\tilde{a}, \tilde{b}) \cap \mathbb{Z}} \Gamma_{\tilde{t}_{0}+\tilde{\varepsilon}}^{\mathbb{Z}}(x)\right] \leqslant \sum_{x \in(\tilde{a}, \tilde{b}) \cap \mathbb{Z}} \mathbb{E}\left[\Gamma_{\tilde{\varepsilon}}^{\mathbb{Z}}(x)\right] \leqslant \frac{C(\tilde{b}-\tilde{a})}{\sqrt{\tilde{\varepsilon}}} \leqslant \frac{C(b-a)}{\sqrt{\varepsilon}} .
\end{aligned}
$$

Strictly speaking, since $\tilde{\varepsilon}=\varepsilon \delta^{-2}$ need not be an integer time, we should estimate $\left|\mathcal{X}_{\delta}^{t_{0}^{-}}\left(t_{0}+\varepsilon\right) \cap(a, b) \times \mathbb{R}\right| \leqslant \sum_{x \in(\tilde{a}, \tilde{b}) \cap \mathbb{Z}}\left(\Gamma_{[\tilde{\varepsilon}]}^{\mathbb{Z}}(x)+\Gamma_{[\tilde{\varepsilon}\rceil}^{\mathbb{Z}}(x)\right)$ but this changes only the constant.

Using Lemma 2.10, Condition $\left(E_{1}^{\prime}\right)$ can then be proved using the strategy from Newman et al. (2005), see Lemma 6.3 there.

\section{Outlook}

Our result can be seen as a convergence result for the space-time embeddings of "all ancestral lines" in a discrete time contact process. More precisely, define the 
contact process as follows: $\left(\eta_{n}^{A}\right)_{n \geqslant m}$ starting at time $m \in \mathbb{Z}$ from the set $A$ as

$$
\eta_{m}^{A}(y)=\mathbb{1}_{A}(y), y \in \mathbb{Z}^{d},
$$

and for $n \geqslant m$

$\eta_{n+1}^{A}(x)= \begin{cases}1 & \text { if } \omega(x, n+1)=1 \text { and } \eta_{n}^{A}(y)=1 \text { for some } y \in \mathbb{Z}^{d} \text { with }\|x-y\| \leqslant 1 \\ 0 & \text { otherwise, }\end{cases}$

i.e., $\eta_{n}^{A}(y)=1$ if and only if there is an open path from $(x, m)$ to $(y, n)$ for some $x \in A$.

By monotonicity, $\mathcal{L}\left(\eta_{n}^{\mathbb{Z}^{d}}\right) \rightarrow \nu$ as $n \rightarrow \infty$, where the convergence is weak convergence and $\nu \in \mathcal{M}\left(\{0,1\}^{\mathbb{Z}^{d}}\right)$ is the upper invariant measure, cf Liggett (1999). Note that the percolation cluster is given as the time-reversal of the stationary process $\eta$. More precisely process $\xi:=\left(\xi_{n}\right)_{n \in \mathbb{Z}}$ defined by $\xi_{n}(x)=\eta_{-n}(x)$, i.e. $\xi_{n}(x)=1$ iff $-\infty \rightarrow^{\omega}(x,-n)$ (defined as $\left.\bigcap_{m \geqslant n}\left\{\mathbb{Z}^{d} \times\{-m\} \rightarrow^{\omega}(x,-n)\right\}\right)$ describes the percolation cluster in the sense that $\xi_{n}(x)=1$ if and only if $x \in \mathcal{C}$. See Birkner et al. (2013) for more details. Hence, the coalescing walkers on the backbone of the cluster correspond to space-time embeddings of all ancestral lines. One may then apply our convergence result to investigate the behaviour of interfaces in the discrete time contact process analogously to Newman et al. (2005, Theorem 7.6 and Remark 7.7). For the continuous-time contact process, interfaces and their scaling limits were analyzed in Mountford and Valesin (2016); Valesin (2010) (without explicitly using a Brownian web limit).

As noted in Remark 1.2, Theorem 1.1 is an "annealed" limit theorem and it would be interesting to prove an analogous "quenched" result. Since Lemma 2.4 is a key ingredient in the proof, we this would require a quenched analogue of (2.11). In this direction, we conjecture (based on simulations) that in $d=1$,

$$
\lim _{n \rightarrow \infty} \sqrt{n} P_{\omega}\left(T_{m e e t}^{\left(z_{1}, z_{2}\right)}>n\right)
$$

exists for $\mathbb{P}$-a.a. $\omega$ (and is a non-trivial function of $\omega$ ).

Acknowledgements. The authors would like to thank Rongfeng Sun for his many helpful comments on the manuscript. We also thank an anonymous referee whose suggestions helped to improve the presentation. M.B. and S.S. were supported by DFG priority programme SPP 1590 through grants BI 1058/3-1 and BI 1058/3-2, N.G. through grant GA 582/7-2.

\section{Appendix A. Proof that $f$ from (2.17) is superharmonic for $\widehat{\Psi}_{\mathrm{diff}}^{\text {joint }}$}

Consider $x>0$, say.

$$
\begin{aligned}
\sum_{y \in \mathbb{Z}} \hat{\Psi}_{\mathrm{diff}}^{\mathrm{joint}}(x, y)(f(y)-f(x)) \leqslant & \sum_{y:|y-x| \geqslant x / 3} \widehat{\Psi}_{\mathrm{diff}}^{\mathrm{joint}}(x, y)(c|y|+c|x|) \\
& +\sum_{y:|y-x|<x / 3} \widehat{\Psi}_{\mathrm{diff}}^{\text {joint }}(x, y)(f(y)-f(x))
\end{aligned}
$$

The first term on the right-hand side is bounded by $C_{2} e^{-c_{2} x}$ for suitable $c_{2}, C_{2} \in$ $(0, \infty)$ by $(2.8)$, for the second term we use Taylor expansion to write it with some 


$$
\begin{aligned}
& \xi_{x, y} \in(x \wedge y, x \vee y) \text { as } \\
& \sum_{y: 0<|y-x|<x / 3} \hat{\Psi}_{\mathrm{diff}}^{\mathrm{joint}}(x, y)\left((y-x) f^{\prime}(x)+\frac{1}{2}(y-x)^{2} f^{\prime \prime}\left(\xi_{x, y}\right)\right)
\end{aligned}
$$

Since $\left|\xi_{x, y}-x\right|<x / 3$ we have $f^{\prime \prime}\left(\xi_{x, y}\right) \leqslant f^{\prime \prime}(4 x / 3)=-2 e^{-4 c_{1} x / 3} \exp \left(2 e^{-4 c_{1} x / 3} / c_{1}\right)$ and thus

$$
\begin{aligned}
& \sum_{y: 0<|y-x|<x / 3} \widehat{\Psi}_{\text {diff }}^{\text {joint }}(x, y) \frac{1}{2}(y-x)^{2} f^{\prime \prime}\left(\xi_{x, y}\right) \\
& \leqslant-2 e^{-4 c_{1} x / 3} \exp \left(2 e^{-4 c_{1} x / 3} / c_{1}\right) \sum_{y: 0<|y-x|<x / 3} \widehat{\Psi}_{\text {diff }}^{\text {joint }}(x, y) \frac{1}{2}(y-x)^{2} \\
& \leqslant-\frac{\tilde{\sigma}^{2}}{2} e^{-4 c_{1} x / 3} \exp \left(2 e^{-4 c_{1} x / 3} / c_{1}\right)
\end{aligned}
$$

(recall (2.16)).

Furthermore, by Lemma 2.2 and (2.8),

$$
\begin{aligned}
& \sum_{y: 0<|y-x|<x / 3} \hat{\Psi}_{\text {diff }}^{\text {joint }}(x, y)(y-x) \\
& \quad \leqslant C_{3} e^{-c_{3} x}+\sum_{y: 0<|y-x| \geqslant x / 3} \widehat{\Psi}_{\text {diff }}^{\text {joint }}(x, y)|y-x| \leqslant C_{4} e^{-c_{4} x}
\end{aligned}
$$

for suitable $c_{3}, C_{3}, c_{4}, C_{4} \in(0, \infty)$,

Combining, we see that the right-hand side of (A.1) is negative if we choose $x_{0}>0$ so large and $c_{1}>0$ so small that (note $f^{\prime}(x)=\exp \left(2 e^{-c_{1} x} / c_{1}\right)$ )

$$
C_{2} e^{-c_{2} x}+\exp \left(2 e^{-c_{1} x} / c_{1}\right) C_{4} e^{-c_{4} x}-\frac{\tilde{\sigma}^{2}}{2} e^{-4 c_{1} x / 3} \exp \left(2 e^{-4 c_{1} x / 3} / c_{1}\right)<0
$$

holds for all $x \geqslant x_{0}$.

\section{References}

M. Abramowitz and I. A. Stegun. Handbook of mathematical functions with formulas, graphs, and mathematical tables, volume 55 of National Bureau of Standards Applied Mathematics Series. For sale by the Superintendent of Documents, U.S. Government Printing Office, Washington, D.C. (1964). MR0167642.

K. S. Alexander. Excursions and local limit theorems for Bessel-like random walks. Electron. J. Probab. 16, no. 1, 1-44 (2011). MR2749771.

R. A. Arratia. Coalescing Brownian motions on the line. Ph.D. thesis, The University of Wisconsin - Madison (1979). MR2630231.

L. Avena, F. den Hollander and F. Redig. Law of large numbers for a class of random walks in dynamic random environments. Electron. J. Probab. 16, no. 21, 587-617 (2011). MR2786643.

S. A. Bethuelsen and F. Völlering. Absolute continuity and weak uniform mixing of random walk in dynamic random environment. Electron. J. Probab. 21, Paper No. 71, 32 (2016). MR3580037.

M. Birkner, J. Černý and A. Depperschmidt. Random walks in dynamic random environments and ancestry under local population regulation. Electron. J. Probab. 21, Paper No. 38, 43 (2016). MR3508685. 
Brownian web limit for coalescing random walks on an oriented percolation cluster 1053

M. Birkner, J. Černý, A. Depperschmidt and N. Gantert. Directed random walk on the backbone of an oriented percolation cluster. Electron. J. Probab. 18, no. 80, 35 (2013). MR3101646.

D. Denisov, D. Korshunov and V. Wachtel. At the Edge of Criticality: Markov Chains with Asymptotically Zero Drift. ArXiv Mathematics e-prints (2016). arXiv: 1612.01592 .

R. Durrett. Oriented percolation in two dimensions. Ann. Probab. 12 (4), 999-1040 (1984). MR757768.

A. Etheridge. Evolution in fluctuating populations. In Mathematical statistical physics, pages 489-545. Elsevier B. V., Amsterdam (2006). MR2581892.

A. M. Etheridge. Survival and extinction in a locally regulated population. Ann. Appl. Probab. 14 (1), 188-214 (2004). MR2023020.

L. R. G. Fontes, M. Isopi, C. M. Newman and K. Ravishankar. The Brownian web: characterization and convergence. Ann. Probab. 32 (4), 2857-2883 (2004). MR2094432.

N. Fournier and S. Méléard. A microscopic probabilistic description of a locally regulated population and macroscopic approximations. Ann. Appl. Probab. 14 (4), 1880-1919 (2004). MR2099656.

G. Grimmett and P. Hiemer. Directed percolation and random walk. In In and out of equilibrium (Mambucaba, 2000), volume 51 of Progr. Probab., pages 273-297. Birkhäuser Boston, Boston, MA (2002). MR1901958.

M. R. Hilário, F. den Hollander, R. S. dos Santos, V. Sidoravicius and A. Teixeira. Random walk on random walks. Electron. J. Probab. 20, no. 95, 35 (2015). MR3399831.

C. Howitt. Stochastic flows and sticky Brownian motion. Ph.D. thesis, University of Warwick (2007).

C. Howitt and J. Warren. Consistent families of Brownian motions and stochastic flows of kernels. Ann. Probab. 37 (4), 1237-1272 (2009). MR2546745.

M. Kimura. Stepping stone model of population. Annual Report of the National Institute of Genetics Japan 3, 62-63 (1953).

D. A. Levin, Y. Peres and E. L. Wilmer. Markov chains and mixing times. American Mathematical Society, Providence, RI (2009). ISBN 978-0-8218-4739-8. MR2466937.

T. M. Liggett. Stochastic interacting systems: contact, voter and exclusion processes, volume 324 of Grundlehren der Mathematischen Wissenschaften [Fundamental Principles of Mathematical Sciences]. Springer-Verlag, Berlin (1999). ISBN 3-540-65995-1. MR1717346.

M. Menshikov, S. Popov and A. Wade. Non-homogeneous random walks. Lyapunov function methods for near-critical stochastic systems, volume 209 of Cambridge Tracts in Mathematics. Cambridge University Press, Cambridge (2017). ISBN 978-1-107-02669-8. MR3587911.

T. Mountford and D. Valesin. Functional central limit theorem for the interface of the symmetric multitype contact process. ALEA Lat. Am. J. Probab. Math. Stat. 13 (1), 481-519 (2016). MR3519255.

C. M. Newman, K. Ravishankar and R. Sun. Convergence of coalescing nonsimple random walks to the Brownian web. Electron. J. Probab. 10, no. 2, 21-60 (2005). MR2120239. 
M. Salvi and F. Simenhaus. Random walk on a perturbation of the infinitely-fast mixing interchange process. J. Stat. Phys. 171 (4), 656-678 (2018). MR3790156.

A. Sarkar and R. Sun. Brownian web in the scaling limit of supercritical oriented percolation in dimension $1+1$. Electron. J. Probab. 18, no. 21, 23 (2013). MR3035749.

E. Schertzer and R. Sun. Perturbations of supercritical oriented percolation and sticky brownian webs. ArXiv Mathematics e-prints (2018). arXiv: 1811.01849.

E. Schertzer, R. Sun and J. M. Swart. Stochastic flows in the Brownian web and net. Mem. Amer. Math. Soc. 227 (1065), vi+160 (2014). MR3155782.

E. Schertzer, R. Sun and J. M. Swart. The Brownian web, the Brownian net, and their universality. In Advances in disordered systems, random processes and some applications, pages 270-368. Cambridge Univ. Press, Cambridge (2017). MR3644280.

S. Steiber. Ancestral lineages in the contact process: scaling and hitting properties. Ph.D. thesis, Johannes Gutenberg-Universität Mainz (2017). https://publications.ub.uni-mainz.de/theses/frontdoor.php? source_opus $=100001057$.

R. Sun. Convergence of coalescing nonsimple random walks to the Brownian Web. Ph.D. thesis, New York University (2005). MR2706830.

B. Tóth and W. Werner. The true self-repelling motion. Probab. Theory Related Fields 111 (3), 375-452 (1998). MR1640799.

D. Valesin. Multitype contact process on $\mathbb{Z}$ : extinction and interface. Electron. J. Probab. 15, no. 73, 2220-2260 (2010). MR2748404.

H. M. Wilkinson-Herbots. Genealogy and subpopulation differentiation under various models of population structure. J. Math. Biol. 37 (6), 535-585 (1998). MR1664797. 